\title{
Seniority based Energy Renormalization Group ( $\Omega$-ERG) Approach in Quantum Chemistry: Initial Formulation and Application to Potential Energy Surfaces
}

\author{
Laimutis Bytautas ${ }^{1, *}$ and Jorge Dukelsky ${ }^{2)}$ \\ 1) Department of Chemistry, Galveston College, 4015 Av. Q, Galveston, TX 77550, USA \\ *Email:1bytauta@gc.edu \\ 2) Instituto de Estructura de la Materia, CSIC, Serrano 123, E-28006 Madrid, Spain
}

\begin{abstract}
This investigation combines the concept of the seniority number $\Omega$ (defined as the total number of singly occupied orbitals in a determinant) with the energy renormalization group (ERG) approach to obtain the lowest-energy electronic states on molecular potential energy surfaces. The proposed $\Omega$-ERG method uses Slater determinants that are ordered according to seniority number $\Omega$ in ascending order. In the $\Omega$-ERG procedure, the active system consists of $M$ (N-electron) states and $K$ additional complement ( $\mathrm{N}$-electron) states (complement-system). Among the $M$ states in the active system the lowest-energy $m$ states represent target states of interest (target-states), thus $m \leq M$. The environment consists of Full Configuration Interaction (FCI) determinants that represent a reservoir from which the complement-states $K$ are being selected. The goal of the $\Omega$-ERG procedure is to obtain lowest-energy target states $m$ of FCI quality in an iterative way at a reduced computational cost. In general, the convergence rate of $\Omega$-ERG energies towards FCI values depends on $m$ and $M$, thus, the notation $\Omega$ $\operatorname{ERG}(m, M)$ is used. It is found that the $\Omega-\operatorname{ERG}(m, M)$ method can be very effective for calculating lowestenergy $m$ (ground and excited) target states when a sufficiently large number of sweeps is used. We find that the fastest convergence is observed when $M>m$. The performance of the $\Omega-\operatorname{ERG}(m, M)$ procedure in describing strongly correlated molecular systems has been illustrated by examining bond-breaking processes in $\mathrm{N}_{2}, \mathrm{H}_{8}, \mathrm{H}_{2} \mathrm{O}$ and $\mathrm{C}_{2}$. The present, proof-of-principle study yields encouraging results for calculating multiple electronic states on potential energy surfaces with near Full CI quality.
\end{abstract}

Keywords: Ab initio Quantum Chemistry; Electronic Structure; Configuration Interaction; Potential Energy Surfaces; Excited States. 


\section{Introduction.}

The development of efficient and reliable many-body approaches focusing on a problem of electron correlation is of great importance in physics and chemistry [1-4]. Density functional theory (DFT) has demonstrated remarkable success [5] in describing equilibrium geometries (local minima on potential energy surfaces) and associated properties of many diverse molecular systems, e.g. organometalic molecules [6] as well as organic magnets [7-9]. One of the major challenges for $a b$ initio methods is calculation of potential energy surfaces for ground and excited electronic states [10]. Despite significant progress much work still needs to be done in this area. There are two considerations when pursuing the development of electron correlation theories, one focuses on strong (or static) correlation, and another on weak (or dynamic) electron correlation (see e.g. ref. [11]). While the first step focuses on zeroth-order wave functions capable of describing strong correlations, the improvement of such wave functions to describe dynamical electron correlation is essential if high-accuracy in molecular properties is required. For instance, a typical case requiring the inclusion of dynamical correlation is calculation of potential energy surfaces capable of yielding near-spectroscopic accuracy in computed rotational-vibrational energy levels [12-23]. The description of strong correlations relies on a properly chosen zeroth-order wave function. Such wave function can serve as a good reference function to include finer correlation effects associated with dynamical correlation in a systematic way. Most frequently, when highly correlated wave functions are sought, a reference function consists of a small expansion of most important Slater determinants. The reference function is usually defined in terms of strongly-occupied molecular orbitals referred to as Fully Optimized Reaction Space (FORS) [24,25] or Complete Active Space (CAS) [26]. The selection of active space in multi-configurational wave functions is a very important topic and has been recently discussed in literature (see, e.g. refs. [27,28]). The Full Configuration Interaction (FCI) approach $[1,2,29]$ yields the exact solution (for a given basis set) and addresses both static and dynamic correlation problems, simultaneously. A considerable effort has been focused to develop computationally efficient algorithms for obtaining FCI quality wave functions and energies (see, e. g. refs. [2,4,30-34]). Since FCI approach is associated with exponential computational scaling, much research has been focused on the development of less computationally demanding ab initio methods for strongly correlated molecular systems. Examples of such methods include: 
truncated (limited) CI methods [35-62] and CI methods with various extrapolations [63-74], multireference coupled cluster (CC) methods [75-80], iterative variational approaches [81-86], various methods based on density-matrices [87-90], matrix product states (MPS) and tensor product states (TPS)-based approaches [91-95] which include popular density-matrix renormalization group (DMRG) method [96-109], tree tensor networks [110], nonlinear wave functions [111-116], various hybrid methods combining coupled-cluster and MPS [117], different versions of CC that capture strong correlation [118-121], Spin-Flip approaches [122,123], methods based on symmetry breaking and restoration [124-126], methods based on Valence Bond-approaches [127], Quantum Monte Carlo approaches [128-131] and many others. In addition, we note that quantum information theory has been found useful in providing conceptual understanding of bond-forming and bond-breaking processes [132].

The concept of seniority, recently borrowed from nuclear physics [133-135], is expressed in terms of the quantum number $\Omega$ [53]. It has been found very useful in earlier investigations $[53,54,136-140]$ in providing a compact description of electron correlation. In our earlier studies $[53,54]$, the seniority number $\Omega$ was used to explore novel CI-selection schemes where criteria for excluding configurations in truncated CI-expansions are determined using criteria based on $\Omega$. Such $\Omega$-truncated CI-expansions along with orbital-optimization procedure have been shown $[53,54]$ to offer a promising avenue for efficiently describing electron correlation in strong correlation regime, e.g. in molecular dissociations. In this study, however, we use the seniority number $\Omega$ to order Slater determinants in Full CI expansion (without truncation) for the use in a renormalization group $(\mathrm{RG})$ procedure. Thus, in the $\mathrm{RG}$ procedure all configurations from the Full CI expansion are effectively considered. In particular, here we use $\Omega$ in the context of the energy renormalization group (ERG) approach of Wilson [141,142]. We note that the energy renormalization group (also called numerical renormalization group) methodology is focused on obtaining the lowest-energy states of ab initio Hamiltonians (see, e.g. refs. [105,143,144]. Thus, the present approach is called the $\Omega$-ERG method. In this proof of principle investigation it is shown that the $\Omega$-ERG method is capable of representing $\mathrm{N}$-electron wave functions for ab initio quantum chemical Hamiltonian in a compact way. The performance of the $\Omega-E R G$ procedure in describing strongly correlated molecular systems has been illuminated by examining bondbreaking processes in $\mathrm{N}_{2}, \mathrm{H}_{8}, \mathrm{H}_{2} \mathrm{O}$ and $\mathrm{C}_{2}$. We note that while iterative approaches for describing electron correlation have been already discussed in literature (see e.g. refs. [81-86]), in most 
cases, the main focus is on the ground electronic state of ab initio Hamiltonian. For instance, the study in ref. [86] explored ground state of $\mathrm{H}_{2} \mathrm{O}$ at several stretched geometries and discussed extensions for describing the first two states at equilibrium geometry of the $\mathrm{H}_{2} \mathrm{O}$ molecule. In other studies, iterative CI-methods have been applied for describing many low-lying states of quantum systems using simplified (not ab initio quantum mechanical) Hamiltonian, like in the case of jellium model for $\mathrm{Na} 8$ metal cluster at fixed equilibrium geometry [85]. For a nice overview of existing iterative approaches the reader is referred to ref. [86]. The $\Omega$-ERG method presented in this work is based on the energy renormalization group approach and seniority number concept and is capable of describing many low-lying states of ab initio quantum chemical Hamiltonian along non-equilibrium geometries yielding FCI-quality results for a given basis set. In this context, we would like to mention that a different-type of RG approach, namely the density-matrix-renormalization-group (DMRG) method pioneered by White [96] has been also very successful for describing strong correlation of ground electronic states. However, in DMRG the criterion for the iterative truncation procedure is based on the reduced density matrix rather than in energy [96-110].

\section{COMPUTATIONAL DETAILS.}

Calculations for $\mathrm{N}_{2}$ (STO-3G basis set), $\mathrm{H}_{8}$ (STO-3G basis set) and $\mathrm{H}_{2} \mathrm{O}$ (3-21G and 6$31 \mathrm{G}$ basis sets) and $\mathrm{C}_{2}$ (6-31G basis sets) have been performed along symmetric dissociation paths. RHF, MCSCF and FCI computations have been carried out by one of us (LB) using the GAMESS quantum chemistry package [145]. The $\Omega-E R G(m, M)$ computations have been carried out by one of us (LB) using in-house pilot code with integral files generated by GAMESS programs [145].

\section{The $\Omega$-ERG method}

The $\Omega$-ERG procedure uses Slater determinants of the Full CI expansion that are ordered in a systematic way where more important determinants (i.e. determinants that have larger weight in a wave function) are included in the $\Omega$-ERG procedure before determinants that are less important 
(i.e. determinants that have lower weight in a wave function). It is possible to estimate the weight of a determinant or configuration a priori and generate CI expansions where Slater determinants are sorted based on a specific procedure [43] while making use of low-substitution-level determinants and exploring the sparsity $[41,42]$ of the FCI wave function. Other methods that are similar in this context can also be found in literature [2,35-37]. Recently, Evangelista and coworkers introduced a method called adaptive configuration interaction (ACI) approach [48,49] for constructing simple multiconfigurational wave functions specified uniquely by an energy cutoff. However, in the present study we use a straightforward and systematic way to order determinants according to their seniority number $\Omega$. This choice is based on our previous findings $[53,54]$ indicating that a balanced description of potential energy surfaces (PES) can be achieved when determinants corresponding to lowest- $\Omega$ values are considered first, and a gradual improvement of such wave functions is achieved when configurations corresponding to higher $\Omega$ values are added in a consecutive way. Furthermore, within each seniority sector, the order of construction of configurations also depends on a list of ordered molecular orbitals. In this study we use Hartree-Fock orbitals that are ordered according to their orbitals energies or natural orbital [146] occupation numbers unless explicitly stated otherwise. However, in general, other choices of MOs can also be utilized, e.g. split-localized MOs [42] or QUAMBOs [147,148]. Finally, within each seniority sector the order in which determinants are generated depends on exact algorithm of construction. Thus, the proposed $\Omega$-ERG method uses Slater determinants that are ordered according to seniority number $\Omega$ in ascending order. In the $\Omega$-ERG procedure, the active system consists of $M$ ( $N$-electron) states and $K$ additional complement ( $N$-electron) states (complement-system). Among $M$ states in the active system, the lowest-energy $m$ states represent target states of interest (target-states), thus $m \leq M$. On the other hand, the environment consists of Full Configuration Interaction (CI) determinants that represent a reservoir from which the complement-states $K$ are being selected. The goal of the $\Omega$-ERG procedure is to obtain lowestenergy target states $m$ of FCI-quality at a reduced computational cost in an iterative fashion. In general, the convergence rate of the $\Omega$-ERG procedure towards the FCI result depends on $m$ and $M$, thus, the notation $\Omega-\operatorname{ERG}(m, M)$ is used. At each iteration, the resulting (small) Hamiltonian matrix of dimension $(M+K) \times(M+K)$ is diagonalized and only $M$ lowest-energy roots (states) are kept for next iteration when $K^{\prime}$ determinants are added from the environment list to form a new 
Hamiltonian matrix of dimension $\left(M+K^{\prime}\right) \times\left(M+K^{\prime}\right)$. In general, the numerical value of $K$ depends on the value of seniority-number $\Omega$ of determinants that are being added and therefore, it will depend on the specific iteration. A higher seniority number $\Omega$ implies that a larger number of $\alpha / \beta$ electron-spin couplings need to be considered for a given set of singly-occupied MOs. Note that in all cases considered in this study the number of $\alpha$-spin electrons is equal to the number of $\beta$-spin electrons. A sweep is completed after all determinants from the Environment (Full CI expansion) had been used in the $\Omega$-ERG procedure. After the first sweep is complete, the $\Omega$-ERG $(m, M)$ procedure is repeated using $M$ lowest-energy states from sweep-one and adding additional $K$ determinants from the Environment. The iterative process is repeated with increasing number of sweeps until satisfactory convergence in energy for all $m$-target states is achieved. It is found that the $\Omega$-ERG $(m, M)$ method can be very effective for calculating the lowest-energy $m$ target (ground and excited) states approaching Full CI result as the number of sweeps increases, meaning that the method is numerically exact. In this study we focus on molecular orbital ordering, number of target states $m$ and number of lowest energy states kept after each diagonalization $(M)$ in order to explore how these factors affect rates of convergence towards exact $N$-electron eigenstates of quantum mechanical Hamiltonian. The performance of the $\Omega$-ERG $(m, M)$ procedure in describing strongly correlated molecular systems is illustrated by examining bond-breaking process in $\mathrm{N}_{2}, \mathrm{H}_{8}, \mathrm{H}_{2} \mathrm{O}$ and $\mathrm{C}_{2}$ (See Table 1 for information regarding $\mathrm{CI}$ wave functions for these systems). The spatial symmetry representing a totally-symmetric irreducible representation of a point group corresponding to a molecular geometry is maintained for all wave functions considered in this study.

\section{A. Definitions relevant to the $\Omega$-ERG $(m, M)$ procedure}

Here we introduce the relevant definitions:

(A) Active System: a set of $M$ determinants (states) that will be included in the construction of the Hamiltonian matrix $\mathbf{H}(\mathbf{i}, \mathbf{j})$. Note that lowest energy $M$-states will be kept after each iteration of the $\Omega$-ERG $(m, M)$ procedure for use in next iteration. 
(B) Complement System: a set of $K$ determinants (see Table 1 for $K$ value for each $\Omega$-sector) from the Environment (see below), which in addition to the $M$ determinants form the Active System that will be used in the construction of the Hamiltonian matrix $\mathbf{H}(\mathbf{i}, \mathbf{j})$. Thus, at each iteration the Hamiltonian matrix has a dimension of $(M+K) \times(M+K)$. We note that the $K$-values in the $\Omega$-ERG $(m, M)$ implementation used in this study are linked to the magnitude of the seniority number $\Omega$ as shown in Table 1. However, the parameter $K$ can be fixed at a relatively small value for all $\Omega$-sectors if desired. For instance, for larger systems and seniorities it will be necessary to partition parameter $K$ to keep dimensions of matrices that are diagonalized in the ERG procedure small.

(C) Environment: a reservoir from which determinants are selected and added to the

Complement System (see above) in each iteration. In this study, except for the first-sweep, the Environment contains a complete set of Full CI determinants. In the first sweep, the first $M$ determinants (in the $\Omega=0$ sector) from the FCI list are chosen to represent Active System. Note that after each iteration new determinants, i.e. determinants that have not been used in the formation of the Hamiltonian matrix in current sweep are selected from the Environment to be added to the Complement System.

(D) Target states: a set of lowest-energy $m$ solutions $(m \leq M)$ that are sought in the $\Omega-\mathrm{ERG}(m, M)$ procedure.

\section{B. The Energy Renormalization Group $\Omega-\operatorname{ERG}(m, M)$ procedure}

Step-1) Formation of ordered set of Full CI determinants. The determinants are ordered according to $\Omega$-sectors in ascending order, namely $\Omega=0,2,4,6$, . and so on. For a list of ordered determinants within each $\Omega$-sector see an illustration of $\mathrm{N}_{2}$ (STO-3G basis set) in the Supplementary material.

See Table 2 for the ordering of determinants in the $\Omega=0$ case $\left(\mathrm{N}_{2}\right.$, STO-3G basis set). 
Step-2) Formation of the Hamiltonian matrix $\mathbf{H}(\mathbf{i}, \mathbf{j})$ for the first iteration of the $\Omega$-ERG $(m, M)$ procedure.

Construct the Hamiltonian matrix of the dimension of $(M+K) \times(M+K)$ using $K+M$ ordered CI-determinants. Note that $K$ value depends on $\Omega$ (see Table I).

Step-3) First iteration: Diagonalization of the Hamiltonian matrix $\mathbf{H}(\mathbf{i}, \mathbf{j})$ of dimension $(M+K) \times(M+K)$.

After the diagonalization is complete, the $M$ lowest-energy roots are kept, while $K$ highest-energy roots are omitted from further consideration.

Step-4) Formation of new Hamiltonian matrix $\mathbf{H}(\mathbf{i}, \mathbf{j})$ for the next iteration of the $\Omega$-ERG $(m, M)$ procedure.

Construct the Hamiltonian matrix of the reduced $(M+K) \times(M+K)$ dimensions using $M$ states kept from the previous iteration and adding $K$ new CI-determinants from the Environment (these $K$ determinants represent the new Complement System).

Step-5) Repeat Steps 2 to 4 until the full list of Full CI determinants from the Environment is exhausted.

This completes the first $\Omega$-ERG $(m, M)$ sweep.

Step-6) Now, consider only $M$ lowest energy states resulting from the first $\Omega$-ERG $(m, M)$ sweep representing a new Active System. Add $K$ determinants from the ordered set of Full CI list of determinants in the Environment.

(Note that these are the same determinants used in the first sweep).

Since the $M$ states and these $K$ determinants are not linearly independent, orthonormalize the set of $(M+K)$ states which requires formation and diagonalization of the overlap matrix $\mathbf{S}(\mathbf{i}, \mathbf{j})$.

Any states that have zero-eigenvalue of the overlap matrix are removed.

We use the canonical orthonormalization procedure [1]. After the set of orthonormal states is determined, construct the Hamiltonian matrix $\mathbf{H}(\mathbf{i}, \mathbf{j})$. 
Step-7) Next iteration: Diagonalize of the Hamiltonian matrix $\mathbf{H}(\mathbf{i}, \mathbf{j})$. After the diagonalization is complete, the $M$ lowest-energy roots are kept, while highest-energy roots are omitted from further consideration.

Step-8) Keep $M$ lowest energy states (eigenvectors), and add $K^{\prime}$ new determinants from the Environment (these $K$ ' determinants represent the Complement System). Then, construct the Hamiltonian matrix $\mathbf{H}(\mathbf{i}, \mathbf{j})$ and Overlap matrix $\mathbf{S}(\mathbf{i}, \mathbf{j})$ of $\left(N+K^{\prime}\right) \times\left(N+K^{\prime}\right)$ dimensions using these $N+K^{\prime}$ vectors.

Note that $K^{\prime}$ value depends on $\Omega$ value (see Table 1).

Orthonormalize the set of $M+K$ ' states which requires diagonalization of the overlap matrix $\mathbf{S}(\mathbf{i}, \mathbf{j})$. Any states that have zero-eigenvalue of the overlap matrix are removed to ensure linearly-independent set of $\mathrm{N}$-electron states. After the set of orthonomal states is determined, the Hamiltonian matrix $\mathbf{H}(\mathbf{i}, \mathbf{j})$ is formed.

Step-9) Next iteration: Diagonalize the Hamiltonian matrix $\mathbf{H}(\mathbf{i}, \mathbf{j})$.

After the diagonalization is complete, the $M$ lowest-energy roots are kept, while highest-energy roots (beyond $M$ ) are omitted from further consideration.

Step-10) Repeat Steps 8 and 9 until the complete list of Full CI determinants is exhausted. This completes the latest $\Omega$-ERG $(m, M)$ sweep.

Step-11) Repeat Steps 6 to 10 until energies of the $m$ target states do not change within the given tolerance of $\varepsilon$. This completes the $\Omega-E R G(m, M)$ procedure.

We shall use symbol $\delta$ to represent the deviation of ERG-values from the exact, i.e. FCIbenchmark. A comment regarding the $K$-value shown in Table 1 used in forming the Complement System of $K$ determinants in each iteration of the $\Omega$-ERG $(m, M)$ procedure is appropriate at this point. The calculation of $K$ value for each $\Omega$ is based on the combinatorial formula: 


$$
K=\frac{(M O S) !}{\left(N_{\alpha}\right) ! \times\left(N_{\beta}\right) !}
$$

Where the symbol $(M O S)$ represents a number of singly-occupied molecular orbitals in a set of occupied MOs, $N_{\alpha}$ is the number of singly-occupied-MOs occupied by electrons having $\alpha$-spin and $N_{\beta}$ is the number of singly-occupied-MOs occupied by electrons having $\beta$-spin. For example, if $\mathbf{A}$ and $\mathbf{B}$ are singly-occupied MOs then there will be two possibilities for $\alpha$ and $\beta$ electron-pair: $\{\mathbf{A}(\alpha), \mathbf{B}(\beta)\}$ and $\{\mathbf{A}(\beta), \mathbf{B}(\alpha)\}$, thus $K=2$. An example displaying the full list of determinants and $\alpha$ - and $\beta$-strings associated with them is provided in the Supplementary material for the case of $\mathrm{N}_{2}$. One can expect that the selection of $K$ value as given by Equation (1) should facilitate the convergence of the $\Omega-\operatorname{ERG}(m, M)$ procedure. Again, as mentioned above, the parameter $K$ can be fixed at a constant and relatively small value for all $\Omega$-sectors if desired. Since the numbers of $\alpha$ - and $\beta$-electrons are the same in all cases considered in the present study, one has $M_{s}=0$ for all wave functions. The $\Omega-\operatorname{ERG}(m, M)$ procedure yields $M_{s}=0$ components of pure spin states (singlets, triplets, quintuplets,...etc). In addition, the spatial symmetry representing totallysymmetric irreducible representations of point groups representing molecular geometries is maintained for all wave functions.

A few comments are appropriate at this point. After the first sweep of the $\Omega-\operatorname{ERG}(m, M)$ procedure, the lowest $M$ states are generated as specific linear combinations of determinants. While after the initial sweep these $M$ states may not be optimal, in following sweeps as additional determinants are included, these lowest states are gradually improved producing new $M$ states that are represented by more optimal linear combinations of determinants. Due to flexibility of the iterative procedure when all determinants of FCI expansion are considered in compact way, the $\Omega$-ERG $(m, M)$ procedure is expected to ultimately converge to $M$ exact benchmark (i.e. FCI) energy values. The exceptions can happen when, at early-sweep-stage, among the lowest $m=M$ roots, there is a root (we shall call it root-X) that is an exact solution to a higher-energy FCI state. In other words, root-X happens to be the $L$-th root of FCI solution, where $L>M=m$. If such a situation occurs, then the $\Omega$-ERG $(m, M)$ procedure may not be able to "switch" high-energy root-X with a lower-energy state, simply by increasing the number of ERG sweeps. The remedy to the situation when a high-energy root gets "stuck" in the small space of $m=M$ roots is to increase the value of $M$ while keeping $m$ unchanged, thus having $m<$ 
$M$. By starting a new $\Omega-\operatorname{ERG}\left(m, M^{\prime}\right)$ procedure where $M^{\prime}>m$, one will observe that the root-X of the earlier $\Omega$-ERG $(m, M)$ procedure with $m=M$ which (incorrectly) occurred as $m$-th root will be replaced by the correct, i.e. $m$-th root of the FCI solution. We shall examine such a situation when discussing the symmetric dissociation of $\mathrm{H}_{8}$ later in this study which illustrates limitations of the ERG procedure.

One may also ask: If all FCI determinants are considered in the $\Omega-\operatorname{ERG}(m, M)$ procedure, why ordering of determinants (in terms of ascending seniority numbers) gradually added to the space of $M$ lowest ERG-roots matter at all? The answer to this question has to do with the rate of convergence to the exact FCI benchmarks. Since earlier studies [53,54] indicated that lowseniority sectors provide reliable and balanced description of bond-breaking chemical processes, it is reasonable to surmise that it is helpful to order the determinants in such a way that moreimportant determinants would be considered before determinants that are less important contributors to lowest energy states. In addition, it is important to note that in the ERGprocedure one starts with a very small number, namely $M$ of $\mathrm{N}$-electron states (determinants), that are improved at each iteration by adding $K$ determinants from the Environment and after diagonalization of the Hamiltonian matrix of dimensions $M+K$ by $M+K$, the new, lowest $M$ roots become linear combinations of determinants, and so on. Thus, the quality of the starting $M$ states (determinants) is important for the fastest convergence toward exact lowest-energy FCI-quality states in this iterative procedure.

Finally, a comment needs to be made regarding the size of basis set and, as a consequence the length of FCI expansions considered in this work. As it was mentioned earlier, we intentionally chose relatively small basis sets and FCI expansions, to reduce the cost and time of our ERG calculations. One may wonder if our principal conclusions about the essence of ERG method and its convergence patterns will be less favorable when larger basis sets and FCI expansions are considered. Firstly, one can say that, ultimately, the size of CI Hamiltonian matrix is a more significant factor than the number of iterations. Secondly, to explore the effects of basis set size and/or the FCI expansion size on the rates of convergence of our ERG method, we consider four molecular systems where the size of FCI expansion increases roughly by a factor of 10, namely, 396, 2468, 61441, and 414864. We note that our pilot-code implementation of the ERG procedure is not as efficient as it can be made to be. Also, it is true that actual timings of our ERG procedure to reach exact FCI benchmarks are higher compared 
even to a straightforward application of FCI codes available in standard ab initio quantum chemistry packages. However, our primary goal in this study is to explore the validity of the ERG procedure to find out if and with how many sweeps it converges to benchmark/exact FCI solutions for various selections of parameter $m$ and $M$ values. At the current stage of our pilotcode, it is practical to treat $M$ values up to 50, and $K$ values up to 70 .

\section{Applications of the $\Omega-\operatorname{ERG}(m, M)$ method}

\section{A. $\mathrm{N}_{2}$ potential energy curve}

A challenging case that illustrates a strong correlation problem is dissociation of the $\mathrm{N}_{2}$ molecule which has been used extensively to estimate the performance of new ab initio methods [53,54,97,48,49,138,149-151]. For simplicity, we choose STO-3G basis set. The spatial symmetry of the wave function corresponds to the $A_{g}$ irreducible representation of $D_{2 h}$ point group. Core 1s-electrons are kept doubly-occupied at all times. The valence-electron correlated active space is (10 electrons in 8 orbitals) for Full CI wave function which contains 396 Slaterdeterminants. The CI determinants are ordered according to Seniority $(\Omega)$ sectors $(\Omega=0,2,4,6)$ in ascending order (see Table 1). In particular, $\Omega=0$ sector has 56 determinants, $\Omega=(0+2)$ sectors contain $116 \mathrm{CI}$ determinants, $\Omega=(0+2+4)$ sectors contain $356 \mathrm{CI}$ determinants, and $\Omega=$ $(0+2+4+6)$ sectors contain 396 CI determinants. See Table 2 for ordering of determinants for $\Omega=0$ case.

In exploring the $\mathrm{N}_{2}$ dissociation case, we will focus on: molecular orbital ordering, number of target states $m$ and the number of the lowest energy states kept after each diagonalization $(M)$ in order to examine how these factors affect the rate of convergence towards exact $N$-electron eigenstates of the quantum mechanical Hamiltonian. The number of added $K$ new N-electron states (Slater determinants) to the previously determined (and kept) states $M$ depends on $\Omega$ (see Table 1). We use Restricted-Hartree-Fock (RHF) molecular orbitals ordered according to their orbital energies unless explicitly noted otherwise. The list of the FCI determinants has been ordered according to their $\Omega$ (from 0 to 6). Table 2 illustrates the order of determinants for $\Omega=0$. For the ordering of determinants for $\Omega=0, \Omega=2, \Omega=4$ and $\Omega=6$ cases, the reader is referred to the Supplementary material. Our target in the case of $\mathrm{N}_{2}$ is four lowest- 
energy states (i.e, $m=4$ ) at various internuclear distances. Figure 1 displays graphically these potential energy curves for $\mathrm{N}_{2}$.

First, we focus on the performance of the $\Omega-\operatorname{ERG}(m, M)$ procedure in converging to the exact energy only for the lowest-energy root, i.e. ground-state (GS) with $m=1$ at three internuclear distances, $\mathrm{R}=1.2 \AA$ (near-equilibrium distance for the GS), $\mathrm{R}=1.6 \AA$ and $\mathrm{R}=2.4 \AA$. We choose $M=4$ (four lowest-energy states kept after each iteration). The performance of the $\Omega$-ERG $(m, M)$ procedure is illustrated in Figure $2(m=1$ and $M=4)$ where we plot logarithmic energy error in Hartree (h) versus the number of ERG sweeps. Figure 2 clearly demonstrates that the convergence of the ground-state energy towards its exact value is very rapid for at $1.2 \AA$ and it becomes slightly slower as the internuclear distance increases. For orbital specifications the reader is referred to the Supplementary material. From the data listing natural-orbital (NO) occupation numbers (see the Supplementary material), one can see that while at $\mathrm{R}=1.2 \AA \mathrm{NO}$ occupation numbers are close either to 2 or 0 , by contrast, at $\mathrm{R}=2.4 \AA$ there are four NO-values that are close to 1 , one NO-occupation number of 1.3 and one NO-occupation of 0.7 while other NOs are doubly-occupied indicating a near-dissociation limit approaching separate $(\mathrm{N}+\mathrm{N})$ atomic states.

Next, we examine $m=4$ lowest states at $1.2 \AA, 1.6 \AA$ and $2.4 \AA$ using the $\Omega$-ERG $(m, M)$ procedure with two $M$ choices: $M=4$ and $M=8$ (see Supplementary material for data corresponding to cases $\mathrm{R}=1.2 \AA$ and $\mathrm{R}=1.6 \AA$ ). The most challenging cases for the convergence rate of $\Omega-\operatorname{ERG}(m, M)$ method occur when $m=M$. For example, for $\mathrm{R}=1.6 \AA$ case, the ERG energies converge to exact values within $\delta=10^{-10}$ Hartree as follows: for the $\Omega$-ERG $(m=2, M=2)$ procedure after 14 sweeps for $m=2$ roots, for the $\Omega$-ERG $(m=3, M=3)$ procedure after 12 sweeps for $m=3$ roots, for the $\Omega$-ERG $(m=4, M=4)$ procedure after 24 sweeps for $m=4$ roots, and for the $\Omega$-ERG $(m=5, M=5)$ procedure after 32 sweeps for $m=5$ roots. Even in the $\mathrm{R}=2.4 \AA$ case, the $\Omega$ $\operatorname{ERG}(m=5, M=5)$ procedure yields all lowest $m=5$ roots within $\delta=10^{-10}$ Hartree from exact results after 50 sweeps (see the Supplementary material). The convergence at $1.2 \AA$ (nearequilibrium bond-length) is very rapid for the lowest-energy $m=4$ target states even for the least favorable, $m=M=4$ case. The lowest three roots converge within $\delta<4 \times 10^{-10}$ Hartree from their FCI benchmarks after only 6 sweeps. For the highest (fourth) root the deviation at sweep=6 is still about $\delta \cong 2.0 \times 10^{-5} \mathrm{~h}$, and it reduces to about $\delta \cong 1 \times 10^{-10} \mathrm{~h}$ at sweep $=16$. On the other hand, 
if $M=8$, all four lowest roots converge within $\delta \leq 4.0 \times 10^{-10} \mathrm{~h}$ from FCI benchmarks only after 9 sweeps. For the case $\mathrm{R}=2.4 \AA$ (see Tables 3 and 4) the convergence of the lowest four roots requires more sweeps to achieve the same accuracy compared to cases $R=1.2 \AA$ and $R=1.6 \AA$ due to the fact that one has to deal with a higher-degree of bond-length stretch. For example, This already can be seen in the comparison of two simplest cases, $\Omega$-ERG $(m=2, M=2)$ and $\Omega$ $\operatorname{ERG}(m=2, M=3)$. The graphical display in Figure 3 demonstrates that the convergence towards two lowest states is much faster for $M=3$ case. The $\Omega-\operatorname{ERG}(m=4, M=4)$ procedure focuses on four lowest states and the convergence data is listed in Table 3. While after 18 sweeps the $\Omega$ $\operatorname{ERG}(m=4, M=4)$ procedure yields the lowest two roots within $\delta=2.0 \times 10^{-10} \mathrm{~h}$, the third and fourth roots have deviations of $1.1 \times 10^{-4} \mathrm{~h}$ and $2.6 \times 10^{-3} \mathrm{~h}$, respectively (see Table 3). However, as shown in Table 4, increasing $M$ from 4 to 8 allows to improve the convergence considerably, and in the $\Omega-\mathrm{ERG}(m=4, M=8)$ procedure all four lowest roots converge to within $\delta=1.3 \times 10^{-9} \mathrm{~h}$ only after 24 sweeps. Thus, the data above clearly demonstrate that having $(m<M)$ accelerates the $\Omega$-ERG $(m, M)$ convergence of $m$ lowest roots to its exact benchmark values of FCI calculation, especially at large internuclear distances. More information about the convergence patterns of the $\Omega$-ERG $(m, M)$ method can be found in the Supplementary material.

Overall, the performance of the $\Omega-\operatorname{ERG}(m, M)$ method at various stretched geometries along the $\mathrm{N}_{2}$ dissociation pathway demonstrate that the order of molecular orbitals can affect the ERG convergence rate for $m=M$ cases. However, when $m<M$, the ERG convergence is much less sensitive to MO ordering. Thus, it is advantageous to choose $m<M$ if the most robust and fastest convergence is sought.

\section{B. H8 potential energy curve}

Symmetric dissociation of hydrogen-chains and hydrogen-cubes have been used extensively to test theoretical methods aimed at describing strong correlation [53,138,152-156]. In ref. [53] the symmetric dissociation of the $\mathrm{H}_{8}$ chain in its ground-electronic state was used to explore the usefulness of seniority number in establishing a FCI hierarchy. The study in ref. [53] demonstrated that criterion for truncating CI expansion based on the seniority number $\Omega$ offers a good alternative to electron-substitution-level based truncation schemes in strong correlation 
case. Here, we revisit the symmetric dissociation of the $\mathrm{H}_{8}$ chain and explore performance of the $\Omega$-ERG $(m, M)$ scheme in converging lowest $m$ roots/states at various internuclear distances along symmetric-dissociation curves. We focus on $\mathrm{Ag}_{\mathrm{g}}$ spatial symmetry states (irreducible representations) of the $\mathrm{D}_{2 \mathrm{~h}}$-point group. Figure 4 displays seven lowest potential energy curves calculated at FCI level of theory using RHF orbitals ordered according to their energies. From Figure 4 it can be seen that $\mathrm{R}_{\mathrm{e}}=0.95 \AA$ represents an equilibrium bond-length for equidistant $\mathrm{H}$ $\mathrm{H}$ internuclear separations between adjacent $\mathrm{H}$-atoms in the $\mathrm{H}_{8}$ chain. Thus, in order to explore the strong correlation regime, we focus on internuclear distances that are much larger than $\mathrm{R}_{\mathrm{e}}$. First, we focus on the lowest four roots/states $(m=4)$ calculated at significantly distorted bondlength distance of $\mathrm{R}=1.8 \AA$. In the $\Omega-\operatorname{ERG}(m, M)$ procedure, we order all RHF orbitals according to their energies, and construct Slater determinants ordering them according to their seniority number in ascending fashion $(\Omega=0,2,4,6,8)$. There are 2468 Slater determinants of spatial symmetry $A_{g}$ (see Table 1). For numerical data illustrating performance of the $\Omega$ $\operatorname{ERG}(m, M)$ method at $\mathrm{R}=1.0 \AA, 1.8 \AA$ and $2.4 \AA$ (see the Supplementary material).

The data for intermediate and large inter-nuclear and equidistant separations between neighboring hydrogen atoms along a potential energy curve for symmetric dissociation of the $\mathrm{H}_{8}$ system are displayed graphically in Figures 5 and 6. Figure 5 illustrates the convergence rate for the lowest $m=4$ states at $\mathrm{R}=1.8 \AA$ while Figure 6 shows the convergence of $m=4$ states at $\mathrm{R}$ $=2.4 \AA$ as number of ERG sweeps increases. Figure $\mathbf{5}$ displays total energies for the lowest four target states $(m=4)$ with $M=6$ (left panel) and $M=20$ (right panel) selections for the ERG method. From Figure 5 it is clear that the convergence for the lowest target states in the $\Omega$ $\operatorname{ERG}(m=4, M=20)$ procedure is considerably faster compared to the $\Omega-\operatorname{ERG}(m=4, M=6)$ method. For instance, for the case $\Omega$-ERG $(m=4, M=6)$ at sweep $=15$, the energy errors $\delta$ relative to FCI benchmark are: $0.010 \mathrm{mh}, 0.031 \mathrm{mh}, 0.253 \mathrm{mh}$ and $1.048 \mathrm{mh}$ for the first (ground-state) $\left({ }^{1} \mathrm{~A}_{\mathrm{g}}\right.$ ) second $\left({ }^{3} \mathrm{~A}_{\mathrm{g}}\right)$, third $\left({ }^{1} \mathrm{~A}_{\mathrm{g}}\right)$, and fourth $\left({ }^{3} \mathrm{~A}_{\mathrm{g}}\right)$ roots, respectively. By contrast, for the case $\Omega$ $\operatorname{ERG}(m=4, M=20)$ at sweep $=15$, the energy errors $\delta$ relative to FCI-benchmark are: 0.000158 $\mathrm{mh}, 0.000878 \mathrm{mh}, 0.000206 \mathrm{mh}$ and $0.014935 \mathrm{mh}$ for the first (ground-state), second, third and fourth lowest-roots, respectively. The procedure works also well if the number of target states is larger than 4. For instance, $m=8$ states converge within $\varepsilon=10^{-10} \mathrm{~h}$ tolerance in the $\Omega$ - 
$\operatorname{ERG}(m=8, M=20)$ procedure after 90 sweeps yielding eight lowest states for $\mathrm{R}=1.8 \AA$ case, each within $\delta=10^{-10} \mathrm{~h}$ from benchmark result (see the Supplementary material).

A short comment regarding the question of convergence of the $\Omega-\operatorname{ERG}(m, M)$ procedure for the least favorable case when $m=M$ is appropriate. At near-equilibrium geometry ( $\mathrm{R}=1.0 \AA$ ), we observe that all target states converge with accuracy of $\delta=10^{-10} \mathrm{~h}$ for $\Omega$-ERG $(m=4, M=4)$ and $\Omega$-ERG $(m=6, M=6)$ after 65 sweeps yielding exact lowest states of the FCI calculation (see the Supplementary material). For stretched geometries, e.g. $R=1.8 \AA$, the convergence of the $\Omega$ $\operatorname{ERG}(m, M)$ procedure when $m=M$ is considerably slower (see Table 5). For the $\Omega$ $\operatorname{ERG}(m=4, M=4)$ procedure all four roots converge within $\varepsilon=1 \times 10^{-10}$ Hartree at sweep $=150$, but only the first three roots match exact benchmark values within $\delta=1 \times 10^{-10} \mathrm{~h}$. The highest (fourth) root from the $\Omega-\mathrm{ERG}(m=4, M=4)$ procedure has energy of -3.61280 Hartree which matches state-38 ( $\left.{ }^{9} \mathrm{~A}_{\mathrm{g}}\right)$ of the FCI calculation (see the Supplementary material). Evidently, the root-4 of the $\Omega$-ERG $(m=4, M=4)$ procedure that represents high-spin-multiplicity state does not interact with any state of the environment and is fully contained in the subspace of $m=M=4$ states. The energy for the state-4 of the FCI solution is $-3.78245 \mathrm{~h}$ (see Table 5). Also, the results in Table 5 show that for cases $\Omega-\operatorname{ERG}(m=6, M=6)$ and $\Omega-\operatorname{ERG}(m=8, M=8)$ the highest root, i.e. root- 6 and root- 8 , respectively, does not yield the highest state, i.e. state- 6 and state- 8 of FCI solution, correctly. Once again, the highest converged ERG root in $\Omega-E R G(m=6, M=6)$ or in $\Omega$-ERG $(m=8, M=8)$ procedure matches state- $38\left({ }^{9} \mathrm{Ag}\right)$ of the FCI calculation. In order to obtain the lowest exact states of FCI-calculation correctly using the $\Omega$-ERG $(m, M)$ procedure, a larger $M$ value (i.e. $m<M$ ) must be selected. As can be seen from Table 5, for the procedures $\Omega$ $\operatorname{ERG}(m=4, M=6), \Omega-\mathrm{ERG}(m=4, M=10)$ and $\Omega$-ERG $(m=8, M=20)$, all $m$ lowest energy ERG-roots converge to correct $m$ FCI-solutions, and the convergence is faster for larger $M$ values.

Even more dramatic effect on the convergence rate of the lowest $m=4$ roots in the $\Omega$ $\operatorname{ERG}(m, M)$ procedure due to the increase of $M$ value is seen at internuclear distance of $2.4 \AA$ where total energies of four lowest states are very close to the dissociation limit of eight separate hydrogen atoms (see the Supplementary material for details). The relevant data is displayed graphically in Figure 6 for the following cases: $\Omega$-ERG $(m=4, M=8)$ (left-panel), $\Omega$ $\operatorname{ERG}(m=4, M=12)$ (middle-panel) and $\Omega-\operatorname{ERG}(m=4, M=20$ ) (right-panel). In particular, the increase of $M$ value from 8 to 20 , reduces the largest deviation observed for state -4 from $1.51 \mathrm{mh}$ 
to only $0.20 \mathrm{mh}$ if only 15 sweeps are considered. At sweep=310 of the $\Omega$-ERG $(m=4, M=20)$ procedure the first three roots are converged within tolerance of $\varepsilon=1 \times 10^{-10} \mathrm{~h}$ having deviations from benchmarks within $\delta=1 \times 10^{-10} \mathrm{~h}$. The root -4 has converged within tolerance of $\varepsilon=4 \times 10^{-9} \mathrm{~h}$ with a deviation from benchmark within $\delta=1 \times 10^{-8} \mathrm{~h}$ (see the Supplementary material).

Overall, the data for the symmetric dissociation of the $\mathrm{H}_{8}$ chain indicate that, in general, higher-energy roots require more sweeps to converge within a given accuracy $\varepsilon$ than lower roots. Furthermore, the convergence rate of the $\Omega-\operatorname{ERG}(m, M)$ method can be increased dramatically when option $m<M$ is chosen, which allows higher roots to converge smoothly to the corresponding exact lowest FCI-quality values without them getting stuck at a higher-energy FCI solution.

\section{C. $\mathrm{H}_{2} \mathrm{O}$ symmetric dissociation}

The next example illustrating the performance of the $\Omega-\operatorname{ERG}(m, M)$ method is the symmetric dissociation of the $\mathrm{H}_{2} \mathrm{O}$ molecule which represents a challenging and useful case for testing new $a b$ initio methods (see, e.g. refs. [54,150,151]). In the recent study [54] the symmetric dissociation of $\mathrm{H}_{2} \mathrm{O}$ was considered to explore hybrid truncated-CI wave functions using seniority-number $\Omega$ and substitution/excitation level $\mathrm{EXC}_{\max }$ with respect to a reference function. The experimentally-derived equilibrium geometry for the ground-state of $\mathrm{H}_{2} \mathrm{O}$ is $\mathrm{R}_{\mathrm{e}}(\mathrm{OH})=$ $0.9572 \AA$ and angle $(\mathrm{HOH})=104.52^{\circ}$ (see, e.g. refs. [54,157]). In our calculations we keep the angle $(\mathrm{HOH})=104.52^{\circ}$ fixed and vary only the internuclear distance $\mathrm{R}_{\mathrm{OH}}$. Basis sets 3-21G and 6-31G are used in order to keep computational costs low. The FCI wave function contains 61441 Slater determinants for $\mathrm{A}_{1}$-symmetry states.

As in cases discussed above, we will primarily focus on stretched distances, e.g. $2 R_{e}$ and $3 \mathrm{R}_{\mathrm{e}}$ since wave functions to be computed by the $\Omega-\operatorname{ERG}(m, M)$ procedure contain increasing amounts of static correlation with the increase of the bond length. We use molecular orbitals obtained from 2-state-avergaged MCSCF calculations using Full Valence Space FVS[8,6] in order to treat energetics of ground and excited states in a more balanced way (see the Supplementary material for details). Two-states included in state-averaged calculations are ${ }^{1} \mathrm{~A}_{1}$ (singlet) and ${ }^{3} \mathrm{~A}_{1}$ (triplet). The list of the FCI determinants has been constructed using these MOs and determinants have been ordered according to $\Omega$ from 0 to 8 (see Table 1). The data 
illustrating the rate of convergence of the $\Omega-\operatorname{ERG}(m, M)$ energies for lowest six $(m=6)$ states of $\mathrm{A}_{1}$ symmetry calculated using 3-21G basis sets are displayed graphically in Figure 7 in terms of logarithmic energy error (in hartree) versus the number of RG sweeps. Left and middle panels of Figure 7 represent data at $2 \mathrm{R}_{\mathrm{e}}$ for $\Omega-\operatorname{ERG}(m=6, M=8)$ and $\Omega-\operatorname{ERG}(m=6, M=10)$, respectively, while the right-panel corresponds to the internuclear distance $3 \mathrm{R}_{\mathrm{e}}$ and $\Omega$-ERG $(m=6, M=16)$ case. The data for $R=2 R_{e}$ shows that the most significant acceleration in the ERG convergence is observed for the second lowest (triplet-state) going from $M=8$ to $M=10$. Most significantly, at $\mathrm{R}=3 \mathrm{R}_{\mathrm{e}}$, the $\Omega$-ERG $(m=6, M=16)$ procedure has much faster convergence than for $M=8$ or $M=10$ at shorter distance of $2 \mathrm{R}_{\mathrm{e}}$.

Potential energy curves describing the symmetric stretch in $\mathrm{H}_{2} \mathrm{O}$ for lowest-energy states of $\mathrm{A}_{1}$-symmetry for 6-31G basis sets are displayed in Figure 8. We use the $\Omega$-ERG $(m, M)$ method to calculate total energies at internuclear distances $R_{e}, 2 R_{e}$ and $3 R_{e}$ which are indicated by solid dots in Figure 8. In the $\Omega-\operatorname{ERG}(m, M)$ procedure (6-31G basis set) we use molecular orbitals obtained from 2-state-avergaged MCSCF calculations using Full Valence Space FVS[8,6] for $A_{1}$ as well. FCI determinants (61,441 Slater determinants) have been constructed using these MOs and determinants have been ordered according to their $\Omega$ in ascending order (see Table 1). Since the $\Omega-E R G(m=6, M=16)$ for $3-21 G$ basis set (see Figure 7) demonstrated rapid convergence, here, for $6-31 \mathrm{G}$ basis set we choose the $\Omega-\operatorname{ERG}(m=6, M=16)$ method to examine convergence for lowest six states in terms of logarithmic energy error (in hartree) compared to FCI energy benchmarks as the number of RG sweeps increases. The relevant data is displayed graphically in Figure 9. From left to right cases are: $R_{e}$ (left-panel), $2 R_{e}$ (middlepanel) and $3 R_{e}$ (right-panel). In the $R_{e}$ case one can see that the convergence is very rapid and that the energy error decreases fastest for lower-energy states and is somewhat slower for higherenergy states. For the $2 \mathrm{R}_{\mathrm{e}}$ case, the $\Omega-\operatorname{ERG}(m=6, M=16)$ procedure displays a slightly slower convergence for the six lowest states compared to the $R_{e}$ case. Finally, for the $3 R_{e}$ case, the $\Omega$ $\mathrm{ERG}(m=6, M=16)$ method yields rapid and nearly identical rate of convergence for all six lowest-energy states indicating that better than microHartree accuracy (i.e. $\delta<10^{-6} \mathrm{~h}$ ) is achieved with only five RG sweeps which is very encouraging. For additional information regarding the convergence rates of the ERG procedures as well as converged energy values see the Supplementary material. 


\section{The $C_{2}$ case}

The last example illustrating the performance of the $\Omega-\operatorname{ERG}(m, M)$ method is the $\mathrm{C}_{2}$ molecule. For the basis set 6-31G, the FCI wave function which correlates 8 valence electrons in 16 orbitals contains 414,864 Slater determinants of $\mathrm{Ag}_{\mathrm{g}}$-symmetry. For simplicity, we select the $\Omega$ $\operatorname{ERG}(m=4, M=12)$ procedure at $\mathrm{R}=1.2 \AA$ inter-nuclear geometry which corresponds to nearequilibrium bond-length. For this selection of $M$ value, the largest Hamiltonian matrix that is considered in the ERG procedure is $82 \times 82$. We compare the convergence rate of the $\Omega$ $\mathrm{ERG}(m=4, M=12)$ procedure in terms of numbers of ERG-sweeps with numbers of CI iterations of Full CI code (ALDET) of Ivanic and Ruedenberg [40] that is available in GAMESS Quantum Chemistry suite of programs [145]. For the Full CI code ALDET we choose several CI input specifications, namely, the size of initial CI guess matrix (ICIGM) and maximum number of CI expansion vectors. The relevant data are displayed in Table 6 which lists only deviations from the benchmarks. For the total energy values the reader is referred to the Supplementary material. From Table 6 one observes that the performance of the $\Omega-\operatorname{ERG}(m=4, M=12)$ procedure compares very well with the Full CI code (ALDET) for the size of initial CI guess matrix (ICIGM) of 300 when comparing sweep numbers with CI-iterations. Only when the size of initial CI guess matrix in the Full CI code is set to 3500, the number of CI iterations and the $\Omega$ ERG $(m=4, M=12)$ sweeps at comparable stages yield similar deviations from benchmarks. While the standard (production-stage) Full CI code is significantly faster than the current pilotimplementation of the $\Omega$-ERG procedure, the data shows that high-quality numerical results can be obtained using only very small CI matrices not exceeding $82 \times 82$ while Full CI expansion contains 414,864 Slater determinants. Also, in order to explore performance of the $\Omega-E R G$ procedure at distorted geometries, we examine the $\mathrm{C}_{2}$ molecule at distorted inter-nuclear bondlength of $1.8 \AA$. The relevant data for $\Omega-\operatorname{ERG}(m=3, M=10)$ and $\Omega-\operatorname{ERG}(m=3, M=14)$ procedures are listed in Table 7. The data shows that the convergence rates of total energies with respect to the number of $\Omega$-ERG sweeps at the inter-nuclear distance of $1.8 \AA$ is slightly slower as compared to its performance at the equilibrium geometry of $1.2 \AA$. However, the convergence 
rates for the lowest 3 states still exhibit rapid convergence (for more data, see the Supplementary Information).

Overall, the results presented in the $\mathrm{C}_{2}$ case are very encouraging and can be useful when developing accurate $a b$ initio methods that consider only very small Hamiltonian matrices.

\section{Summary and Conclusions.}

In the present study we have introduced the energy (also called numerical) renormalization group approach, $\Omega$-ERG $(m, M)$ for calculating Full CI energies of $m$ lowest-energy electronic states. In the $\Omega$-ERG $(m, M)$ method, the ordering of Slater determinants is based on the seniority-number $(\Omega)$ in ascending order, i.e. determinants from lower- $\Omega$ sectors are included before higher- $\Omega$ sectors (i.e., $\Omega=0,2,4$, etc). For simplicity, we used small basis sets to test our pilot code of the $\Omega-\mathrm{ERG}(m, M)$ procedure in order to keep the calculations tractable and to keep computational costs low. Also, we focused on small numbers of lowest target states $m$ in most cases $(5 \leq m \leq 8)$ to discuss the performance of the pilot-code of the $\Omega$-ERG $(m, M)$ procedure for dissociation of $\mathrm{N}_{2}$ and $\mathrm{H}_{8}$ and the water molecule. In addition, we calculated lowest states for the $\mathrm{C}_{2}$ molecule at several inter-nuclear distances to see how the increase of the total number of determinants to be included affects the convergence rates in terms of ERG-sweeps. Our investigation shows that for the fastest convergence of the $\Omega-\operatorname{ERG}(m, M)$ method it is beneficial to use $M$ values that are larger than the number of target states $m$, i.e. $M>m$. However, even for the least favorable case of $m=M$ our ERG procedure is able to recover the exact $m$ lowest-energy target eigenvalues with enough number of sweeps for most cases. The only exception occurred when the space of $M$ lowest-states contained a high-spin (multiplicity = 9) state that did not interact with environment states when the selection $m=M$ was made. However, the increase of the $M$ value in the $\Omega$-ERG-procedure enabled the convergence of the all lowest $m$ roots (targetstates) toward correct FCI benchmarks.

The present investigation demonstrates that the $\Omega-\operatorname{ERG}(m, M)$ method is capable of recovering Full CI energies within a chosen accuracy $\varepsilon$, for arbitrarily selected lowest energy states $m$ representing various spin-multiplicities (singlets, triplets, quintuplets, etc.) along potential energy surfaces. One criterion that is relevant when discussing the computational cost 
of the $\Omega$-ERG $(m, M)$ method is the dimension of largest matrix that needs to be diagonalized in the iterative ERG procedure relative to the size of Full CI expansion in terms of Slater determinants. The largest matrices diagonalized in the $\Omega-\operatorname{ERG}(m, M)$ procedure have dimensions of $28 \times 28,90 \times 90,86 \times 86$ and $84 \times 84$ compared to FCI matrix-dimensions of $396 \times 396$, $2468 \times 2468,61441 \times 61441$, and $414864 \times 414864$ for $\mathrm{N}_{2}, \mathrm{H}_{8}, \mathrm{H}_{2} \mathrm{O}$ and $\mathrm{C}_{2}$, respectively. The rate of convergence of the ERG procedure towards benchmark values for lowest energy states is very similar for all these systems indicating that there is no significant deterioration of ERG convergence rates with RG-sweeps while the size of FCI expansion increases dramatically. A further improvement in the performance in the $\Omega$-ERG $(m, M)$ method can be achieved by incorporating protocols and concepts of quantum information theory as described in refs. [100$102,132]$.

Overall, the present proof-of-principle study indicates that the $\Omega$-ERG $(m, M)$ method for determining low-lying states (ground-state and excited states) along the dissociation paths is an effective and promising approach. There are many ways to improve the effectiveness of the iterative $\Omega$-ERG $(m, M)$ method, for example, one way is to select only the most important determinants in the trial expansion. In future applications, the $\Omega-\operatorname{ERG}(m, M)$ method will be tested for other cases of strongly-correlated systems exploring truncated CI-expansions to explore its performance. For instance, the ERG-method can be useful within the context of the CEEIS-FCI method (see refs. [66-70]).

\section{Supplementary material:}

This Supplementary material lists raw data for describing the performance of the $\Omega$-ERG method for $\mathrm{N}_{2}, \mathrm{H}_{8}, \mathrm{H}_{2} \mathrm{O}$ and $\mathrm{C}_{2}$ systems. 


\section{Acknowledgements:}

L.B. thanks Dr. Michael W. Schmidt (Iowa State University) for his continued assistance with the GAMESS package of Quantum Chemistry programs. L.B. gratefully acknowledges the computational resources of the theoretical chemistry group in the Department of Chemistry at Iowa State University. L.B. gratefully acknowledges fruitful discussions with Professor Douglas J. Klein (Texas A\&M University at Galveston). The authors thank Professor Gustavo E. Scuseria (Rice University) for helpful suggestions and stimulating discussions during the early stages of this work. L.B. thanks Professor Klaus Ruedenberg (Iowa State University) for his

interest and stimulating discussions. J.D. is supported by Grant No. FIS2015-63770-P (MINECO/FEDER). 


\section{References:}

[1] A. Szabo, N. S. Ostlund, Modern Quantum Chemistry, McGraw-Hill (1989).

[2] Sherrill, C. D.; Schaefer, III, H. F. The Configuration Interaction Method: Advances in Highly Correlated Approaches. Adv. Quantum Chem. 34, (1999), 143.

[3] C. D. Sherrill, Frontiers in Electronic Structure Theory. J. Chem. Phys. 132, (2010), 110902.

[4] P. G. Szalay, T. Müller, G. Gidofalvi, H. Lischka, R. Shepard, Multiconfiguration Self-consistent Field and Multireference Configuration Interaction Methods and Applications. Chem. Rev. 112, (2012), 108.

[5] W. Kohn, Nobel Lecture: Electronic Structure of Matter-Wave Functions and Density Functional. Rev. Mod. Phys. 71, (1999), 1253.

[6] L. Bytautas, Stability of Dimethylmercury and Related Mercurycontaining Compounds with Respect to Selected Chemical Species Found in Aqueous Environment. Croat. Chem. Acta 86, (2013), 453.

[7] D. Bhattacharya, S. Shil, A. Misra, L. Bytautas, D. J. Klein, Borazine: Spin Blocker or Not? Phys. Chem. Chem. Phys. 17, (2015), 14223.

[8] D. Bhattacharya, S. Shil, A. Misra, L. Bytautas, D. J. Klein, Photomagnetic and Nonlinear Optical Properties in cis-trans Green Fluoroprotein Chromophore Coupled Bis-imino Nitroxide Diradicals. Int. J. Quantum Chem. 115, (2015), 1561. 
[9] D. Bhattacharya, S. Shil, A. Misra, L. Bytautas, D. J. Klein, Toward Molecular Magnets of Organic Origin via Anion- $\pi$ Interaction Involving m-Aminyl Diradical: A Theoretical Study. J. Phys. Chem. A 120, (2016), 9117.

[10] L. Bytautas, J. M. Bowman, X. Huang, A.J.C. Varandas, Accurate Potential Energy Surfaces and Beyond: Chemical Reactivity, Binding, Long-Range Interactions, and Spectroscopy. Adv. Phys. Chem. 2012, (2012), 679869.

[11] C. L. Benavides-Riveros, N. N. Lathiotakis, M. A. L. Marques, Towards a Formal Definition of Static and Dynamic Electronic Correlations. Phys. Chem. Chem. Phys. 19, (2017), 12655.

[12] W. Kolos, L. Wolniewicz, Improved Theoretical Ground-State Energy of the Hydrogen Molecule. J. Chem. Phys. 49, (1968), 404.

[13] R. J. Gdanitz, Accurately Solving the Electronic Schrödinger Equation of Atoms and Molecules using Explicitly Correlated $\left(\mathrm{r}_{12^{-}}\right) \mathrm{MR}-\mathrm{CI}$ : the Ground State Potential Energy Curve of $\mathrm{N}_{2}$. Chem. Phys. Lett. 283, (1998), 253.

[14] K. Patkowski, V. Špirko, K. Szalewicz, On the Elusive Twelfth Vibrational State of Beryllium Dimer. Science 326, (2009), 1382.

[15] S. Sharma, T. Yanai, G. H. Booth, C. J. Umrigar, G. K.-L. Chan, Spectroscopic Accuracy Directly from Quantum Chemistry: Application to Ground and Excited States of Beryllium Dimer. J. Chem. Phys. 140, (2014), 104112. 
[16] L. Bytautas, T. Nagata, M.S. Gordon, K. Ruedenberg, Accurate ab initio Potential Energy Curve of $F_{2}$. I. Nonrelativistic Full Valence Configuration Interaction Energies using the Correlation Energy Extrapolation by Intrinsic Scaling method. J. Chem. Phys. 127, (2007), 164317.

[17] L. Bytautas, N. Matsunaga, T. Nagata, M.S. Gordon, K. Ruedenberg, Accurate ab initio Potential Energy Curve of $F_{2}$. III. The Vibration Rotation Spectrum. J. Chem. Phys. 127, (2007), 204313.

[18] L. Bytautas, K. Ruedenberg, Ab initio Potential Energy Curve of $F_{2}$. IV. Transition from the Covalent to the Van der Waals Region: Competition between Multipolar and Correlation Forces. J. Chem. Phys. 130, (2009), 204101.

[19] L. Bytautas, K. Ruedenberg, Accurate ab initio Potential Energy Curve of O $\mathrm{O}_{2}$. I. Nonrelativistic Full Configuration Interaction Valence Correlation by the Correlation Energy Extrapolation by Intrinsic Scaling Method. J. Chem. Phys. 132, (2010), 074109.

[20] L. Bytautas, N. Matsunaga, K. Ruedenberg, Accurate ab initio Potential Energy Curve of $\mathrm{O}_{2}$. II. Core-valence Correlations, Relativistic Contributions, and Vibration-rotation Spectrum. J. Chem. Phys. 132, (2010), 074307.

[21] L. Bytautas, N. Matsunaga, G. E. Scuseria, K. Ruedenberg, Accurate Potential Energy Curve for $B_{2}$. Ab Initio Elucidation of the Experimentally Elusive Ground State Rotation-Vibration Spectrum. J. Phys. Chem. A 116, (2012), 1717.

[22] B. Csontos, B. Nagy, J. Csontos, M. Kállay, Dissociation of the Fluorine Molecule. J. Phys. Chem. A 117, (2013), 5518.

[23] J. S. Boschen, D. Theis, K. Ruedenberg, T. L. Windus, Accurate ab initio Potential Energy Curves and Spectroscopic Properties of the Four Lowest Singlet States of $C_{2}$. Theor. Chem. Acc. 133, (2014), 1425. 
[24] L. M. Cheung, K. R. Sundberg, K. Ruedenberg, Electronic Rearrangements During Chemical Reactions. II. Planar Dissociation of Ethylene. Int. J. Quantum Chem. 16, (1979), 1103.

[25] K. Ruedenberg, M. W. Schmidt, M. M. Gilbert, S. T. Elbert, Are Atoms Intrinsic to Molecular Electronic Wavefunctions? I. The FORS Model. Chem. Phys. 71, (1982), 41.

[26] B. O. Roos, The Complete Active Space Self-Consistent Field Method and its Applications in Electronic Structure Calculations. Adv. Chem. Phys. 69, (1987), 399.

[27] M. W. Schmidt, M. S. Gordon, The Construction and Interpretation of MCSCF Wavefunctions. Annu. Rev. Phys. Chem. 49, (1998), 233.

[28] S. Keller, K. Boguslawski, T. Janowski, M. Reiher, P. Pulay, Selection of Active Spaces for Multiconfigurational Wavefunctions. J. Chem. Phys. 142, (2015), 244104.

[29] I. Shavitt, The History and Evolution of Configuration Interaction. Mol. Phys. 94, (1998), 3.

[30] E. R. Davidson, The Iterative Calculation of a Few of the Lowest Eigenvalues and Corresponding Eigenvectors of Large Real-symmetric Matrices.

J. Comput. Phys. 17, (1975), 87.

[31] N. C. Handy, Multi-root Configuration Interaction Calculations. Chem. Phys. Lett. 74, (1980), 280. 
[32] P. Knowles, Very Large Full Configuration Interaction Calculations. Chem. Phys. Lett. 155, (1989), 513.

[33] A. O. Mitrushenkov, Passing the Several Billions Limit in FCI Calculations on a MiniComputer. Chem. Phys. Lett. 217, (1994), 559.

[34] Z. Rolik, Á. Szabados, P. R. Surján, A Sparse Matrix Based Full-Configuration Interaction Algorithm. J. Chem. Phys. 128, (2008), 144101.

[35] R. J. Buenker, S. D. Peyerimhoff, Individualized Configuration Selection in CI Calculations with Subsequent Energy Extrapolation. Theor. Chim. Acta 35, (1974), 33.

[36] B. Huron, J. P. Malrieu, P. Rancurel, Iterative Perturbation Calculations of Ground and Excited State Energies from Multiconfigurational Zeroth-order Wavefunctions. J. Chem. Phys. 58, (1973), 5745.

[37] C. F. Bender, E. R. Davidson, Studies in Configuration Interaction: The First-row Diatomic Hydrides. Phys. Rev. 183, (1969), 23.

[38] C. Angeli, R. Cimiraglia, M. Persico, Multireference Perturbation CI III. Fast Evaluation of the One-particle Density Matrix. Theor. Chem. Acc. 100, (1998), 324.

[39] J. Olsen, B. O. Roos, P. Jørgensen, H. J. A. Jensen, Determinant Based Configuration Interaction Algorithms for Complete and Restricted Configuration Interaction Spaces. J. Chem. Phys. 89, (1988), 2185.

[40] J. Ivanic, K. Ruedenberg, Identification of Deadwood in Configuration Spaces through General Direct Configuration Interaction. Theor. Chem. Acc. 106, (2001), 339.

[41] J. Ivanic, K. Ruedenberg, Deadwood in Configuration Spaces. II. Singles+ Doubles and Singles+ Doubles+ Triples+ Quadruples Spaces. Theor. Chem. Acc. 107, (2002), 220. 
[42] L. Bytautas, J. Ivanic, K. Ruedenberg, Split-localized Orbitals can Yield Stronger Configuration Interaction Convergence than Natural Orbitals.

J. Chem. Phys. 119, (2003), 8217.

[43] L. Bytautas, K. Ruedenberg, A priori Identification of Configurational Deadwood. Chem. Phys. 356, (2009), 64.

[44] J. Ivanic, Direct Configuration Interaction and Multiconfigurational Self-consistent-field Method for Multiple Active Spaces with Variable Occupations. I. Method.

J. Chem. Phys. 119, (2003), 9364.

[45] M. L. Abrams, C. D. Sherrill, Important Configurations in Configuration Interaction and Coupled-cluster Wave Functions. Chem. Phys. Lett. 412, (2005), 121.

[46] M. L. Abrams, C. D. Sherrill, On the Orbital Dependence of Compact, Weight-selected Configuration Interaction and Coupled-cluster Wave Functions.

Mol. Phys. 103, (2005), 3315.

[47] G. Li Manni, D. Ma, F. Aquilante, J. Olsen, L. Gagliardi, SplitGAS Method for Strong Correlation and the Challenging Case of $\mathrm{Cr}_{2}$. J. Chem. Theory Comput. 9, (2013), 3375.

[48] F. A. Evangelista, Adaptive Multiconfigurational Wave Functions.

J. Chem. Phys. 140, (2014), 124114.

[49] J. B. Schreiber, F. A. Evangelista, Communication: An Adaptive Configuration Interaction Approach for Strongly Correlated Electrons with Tunable Accuracy. J. Chem. Phys. 144, (2016), 161106.

[50] P. J. Knowles, Compressive Sampling in Configuration Interaction Wavefunctions. Mol. Phys. 113, (2015), 1655. 
[51] R. J. Harrison, Approximating Full Configuration Interaction with Selected Configuration Interaction and Perturbation Theory. J. Chem. Phys. 94, (1991), 5021.

[52] M. Hanrath, B. Engels, New Algorithms for an Individually Selecting MR-CI program. Chem. Phys. 225, (1997), 197.

[53] L. Bytautas, T. M. Henderson, C. A. Jiménez-Hoyos, J. K. Ellis, G. E. Scuseria, Seniority and Orbital Symmetry as Tools for Establishing a Full Configuration Interaction Hierarchy. J. Chem. Phys. 135, (2011), 044119.

[54] L. Bytautas, G. E. Scuseria, K. Ruedenberg, Seniority Number Description of Potential Energy Surfaces: Symmetric Dissociation of Water, $\mathrm{N}_{2}, \mathrm{C}_{2}$, and $\mathrm{Be}_{2}$. J. Chem. Phys. 143, (2015), 094105.

[55] D. R. Alcoba, A. Torre, L. Lain, G. E. Massaccesi, O. B. Oña, Seniority Number in Spin-adapted Spaces and Compactness of Configuration Interaction Wave Functions. J. Chem. Phys. 139, (2013), 084103.

[56] D. R. Alcoba, A. Torre, L. Lain, O. B. Oña, P. Capuzzi, M. Van Raemdonck, P. Bultinck, D. Van Neck, A Hybrid Configuration Interaction Treatment based on Seniority Number and Excitation Scheme. J. Chem. Phys. 141, (2014), 244118.

[57] D. R. Alcoba，A. Torre, L. Lain, G. E. Massaccesi, O. B. Oña, Configuration Interaction Wave Functions: A Seniority Number Approach. J. Chem. Phys. 140, (2014), 234103.

[58] W. Poelmans, M. Van Raemdonck, B. Verstichel, S. De Beardemacker, A. Torre, L. Lain, G. E. Massaccesi, D. R. Alcoba, P. Bultinck, D. Van Neck, Variational Optimization of the Second-order Density Matrix Corresponding to a SeniorityZero Configuration Interaction Wave Function. J. Chem. Theory Comput. 11, (2015), 4064. 
[59] D. R. Alcoba, A. Torre, L. Lain, G. E. Massaccesi, O. B. Oña, P. Capuzzi, Spin Contamination-free N-electron Wave Functions in the Excitation-based Configuration Interaction Treatment. J. Chem. Phys. 145, (2016), 014109.

[60] A. A. Holmes, N. M. Tubman, C. J. Umrigar, Heat-Bath Configuration Interaction: An Efficient Selected Configuration Interaction Algorithm Inspired by Heat-Bath Sampling. J. Chem. Theory Comput. 12, (2016), 3674.

[61] T. Zhang, F. A. Evangelista, A Deterministic Projector Configuration Interaction Approach for the Ground State of Quantum Many-body Systems.

J. Chem. Theory Comput. 12, (2016), 4326.

[62] J. R. McClean, A. Aspuru-Guzik, Compact Wavefunctions from Compressed Imaginary Time Evolution. RSC Adv. 5, (2015), 102277.

[63] C. F. Bunge, Selected Configuration Interaction with Truncation Energy Error and Application to the Ne Atom. J. Chem. Phys. 125, (2006), 014107.

[64] C. X. Almora-Díaz, Highly Correlated Configuration Interaction Calculations on Water with Large Orbital Bases. J. Chem. Phys. 140, (2014), 184302.

[65] C. X. Almora-Díaz, H. I. Rivera-Arrieta, C. F. Bunge, Chapter Six-Recent Progress in the Variational Orbital Approach to Atomic and Molecular Electronic Structure. Adv. Quantum Chem. 72, (2016), 129.

[66] L. Bytautas, K. Ruedenberg, Correlation Energy Extrapolation by Intrinsic Scaling. I. Method and Application to the Neon Atom. J. Chem. Phys. 121, (2004), 10905.

[67] L. Bytautas, K. Ruedenberg, Correlation Energy Extrapolation by Intrinsic Scaling. II. The Water and the Nitrogen Molecule. J. Chem. Phys. 121, (2004), 10919. 
[68] L. Bytautas, K. Ruedenberg, Correlation Energy Extrapolation by Intrinsic Scaling. IV. Accurate Binding Energies of the Homonuclear Diatomic Molecules Carbon, Nitrogen, Oxygen, and Fluorine. J. Chem. Phys. 122, (2005), 154110.

[69] L. Bytautas, K. Ruedenberg, Correlation Energy Extrapolation by Intrinsic Scaling. V. Electronic Energy, Atomization Energy, and Enthalpy of Formation of Water. J. Chem. Phys. 124, (2006), 174304.

[70] L. Bytautas, K. Ruedenberg, The Range of Electron Correlation between Localized Molecular Orbitals. A Full Configuration Interaction Analysis for the NCCN Molecule. J. Phys. Chem. A 114, (2010), 8601.

[71] D. Theis, J. Ivanic, T. L. Windus, K. Ruedenberg, The Transition from the Open Minimum to the Ring Minimum on the Ground State and on the Lowest Excited State of Like Symmetry in Ozone: A Configuration Interaction Study.

J. Chem. Phys. 144, (2016), 104304.

[72] J. S. Boschen, D. Theis, K. Ruedenberg, T. L. Windus, Correlation Energy Extrapolation by Many-Body Expansion. J. Phys. Chem. A 121, (2017), 836.

[73] P. M. Zimmerman; Incremental Full Configuration Interaction. J. Chem. Phys. 146, (2017), 104102.

[74] P. M. Zimmerman, Strong correlation in incremental full configuration interaction. J. Chem. Phys. 146, (2017), 224104.

[75] U. S. Mahapatra, B. Datta, B. Bandyopadhyay, D. Mukherjee, State-specific Multi-reference Coupled Cluster Formulations: Two Paradigms. Adv. Quantum Chem. 30, (1998), 163. 
[76] A. Balková, R. J. Bartlett, A Multireference Coupled-cluster Study of the Ground State and Lowest Excited States of Cyclobutadiene. J. Chem. Phys. 101, (1994), 8972.

[77] J. Meller, J. P. Malrieu, R. Caballol, State-specific Coupled Cluster-type Dressing of Multireference Singles and Doubles Configuration Interaction Matrix. J. Chem. Phys. 104, (1996), 4068.

[78] E. Giner, G. David, A. Scemama, J. P. Malrieu, A Simple Approach to the State-specific MR-CC using the Intermediate Hamiltonian Formalism. J. Chem. Phys. 144, (2016), 064101.

[79] U. S. Mahapatra, B. Datta, D. Mukherjee, A State-specific Multi-reference Coupled Cluster Formalism with Molecular Applications. Mol. Phys. 94, (1998), 157.

[80] I. Hubač, J. Pittner, P. Čársky, Size-Extensivity Correction for the State-specific Multireference Brillouin-Wigner Coupled-cluster Theory. J. Chem. Phys. 112, (2000), 8779.

[81] H. Nakatsuji, M. Ehara, Iterative CI General Singles and Doubles (ICIGSD) Method for Calculating the Exact Wave Functions of the Ground and Excited States of Molecules. J. Chem. Phys. 122, (2005), 194108.

[82] H. Nakatsuji, Full Configuration-Interaction Calculations with the Simplest Iterative Complement Method: Merit of the Inverse Hamiltonian. Phys. Rev. A 84, (2011), 062507.

[83] H. Nakatsuji, Discovery of a General Method of Solving the Schrödinger and Dirac Equations That Opens a Way to Accurately Predictive Quantum Chemistry. Acc. Chem. Res. 45, (2012), 1480.

[84] H. Nakatsuji, H. Nakashima, Solving the Schrödinger Equation of Molecules by Relaxing the Antisymmetry Rule: Inter-exchange Theory. J. Chem. Phys. 142, (2015), 194101. 
[85] M. Sambataro, D. Gambacurta, L. Lo Monaco, Iterative Variational Approach to Finite Many-body Systems. Phys. Rev. B 83, (2011), 045102.

[86] W. Liu, M. R. Hoffmann, iCI: Iterative CI toward Full CI.

J. Chem. Theory Comput. 12, (2016), 1169.

[87] D. A. Mazziotti, Two-electron Reduced Density Matrix as the Basic Variable in Many-electron Quantum Chemistry and Physics. Chem. Rev. 112, (2012), 244.

[88] M. Nakata, M. Ehara, H. Nakatsuji, Density Matrix Variational Theory: Application to the Potential Energy Surfaces and Strongly Correlated Systems.

J. Chem. Phys. 116, (2002), 5432.

[89] G. Knizia, G. K.-L. Chan, Density Matrix Embedding: A Strong-coupling Quantum Embedding Theory. J. Chem. Theory Comput. 9, (2013), 1428.

[90] C. Liu, J. Liu, Y. X. Yao, P. Wu, C. Z. Wang, K. M. Ho, Correlation Matrix Renormalization Theory: Improving Accuracy with Two-electron Density-matrix Sum Rules. J. Chem. Theory Comput. 12, (2016), 4806.

[91] M. Nakatani, G. K.-L. Chan, Efficient Tree Tensor Network States (TTNS) for Quantum Chemistry: Generalizations of the Density Matrix Renormalization Group Algorithm. J. Chem. Phys. 138, (2013), 134113.

[92] S. Szalay, M. Pfeffer, V. Murg, G. Barcza, F. Verstraete, R. Schneider, Ö. Legeza, Tensor Product Methods and Entanglement Optimization for ab initio Quantum Chemistry. Int. J. Quantum Chem. 115, (2015), 1342.

[93] K.-H. Böhm, A. A. Auer, M. Espig, Tensor Representation Techniques for Full Configuration Interaction: A Fock Space Approach using the Canonical Product Format. J. Chem. Phys. 144, (2016), 244102. 
[94] A. Kovyrshin, M. Reiher, Tensor Network States with Three-site Correlators. New J. Phys. 18, (2016), 113001.

[95] L. Veis, A. Antalík, J. Brabec, F. Neese, Ö. Legeza, J. Pittner, Coupled Cluster Method with Single and Double Excitations Tailored by Matrix Product State Wave Functions. J. Phys. Chem. Lett. 7, (2016), 4072.

[96] S. R. White, Density Matrix Formulation for Quantum Renormalization Groups. Phys. Rev. Lett. 69, (1992), 2863.

[97] S. R. White, R. L. Martin, Ab initio Quantum Chemistry using the Density Matrix Renormalization Group. J. Chem. Phys. 110, (1999), 4127.

[98] S. Daul, I. Ciofini, C. Daul, S. R. White, Full-CI Quantum Chemistry using the Density Matrix Renormalization Group. Int. J. Quantum Chem. 79, (2000), 331.

[99] G. Barcza, Ö. Legeza, K. H. Marti, M. Reiher, Quantum-information Analysis of Electronic States of Different Molecular Structures. Phys. Rev. A 83, (2011), 012508.

[100] Ö. Legeza, J. Röder, B. A. Hess, Controlling the Accuracy of the Density-Matrix Renormalization-Group Method: The Dynamical Block State Selection Approach. Phys. Rev. B 67, (2003), 125114.

[101] J. Rissler, R. M. Noak, S. R. White, Measuring Orbital Interaction Using Quantum Information Theory. Chem. Phys. 323, (2006), 519.

[102] H.-G. Luo, M.-P. Qin, T. Xiang, Optimizing Hartree-Fock Orbitals by the Density-Matrix Renormalization Group. Phys. Rev. B 81, (2010), 235129. 
[103] G. K.-L. Chan, M. Head-Gordon, Highly Correlated Calculations with a Polynomial Cost Algorithm: A Study of the Density Matrix Renormalization Group.

J. Chem. Phys. 116, (2002), 4462.

[104] G. K.-L. Chan, M. Kállay, J. Gauss, State-of-the-art Density Matrix Renormalization Group and Coupled Cluster Theory Studies of the Nitrogen Binding Curve.

J. Chem. Phys. 121, (2004), 6110.

[105] J. Dukelsky, S. Pittel, The Density Matrix Renormalization Group for Finite Fermi Systems. Rep. Prog. Phys. 67, (2004), 513.

[106] G. Moritz, M. Reiher, Decomposition of Density Matrix Renormalization Group States into a Slater Determinant Basis. J. Chem. Phys. 126, (2007), 244109.

[107] D. Zgid, M. Nooijen, The Density Matrix Renormalization Group Self-consistent Field Method: Orbital Optimization with the Density Matrix Renormalization Group Method in the Active Space. J. Chem. Phys. 128, (2008), 144116.

[108] Y. Kurashige, G. K.-L. Chan, T. Yanai, Entangled Quantum Electronic Wavefunctions of the $\mathrm{Mn}_{4} \mathrm{CaO}_{5}$ Cluster in Photosystem II. Nat. Chem. 9, (2013), 660.

[109] Y. Ma, J. Wen, H. Ma, Density-matrix Renormalization Group Algorithm with Multi-level Active Space. J. Chem. Phys. 143, (2015), 034105.

[110] V. Murg, F. Verstraete, Ö. Legeza, R. M. Noack, Simulating Strongly Correlated Quantum Systems with Tree Tensor Networks. Phys. Rev. B 82, (2010), 205105.

[111] R. Shepard, A General Nonlinear Expansion form for Electronic Wave Functions. J. Phys. Chem. A 109, (2005), 11629.

[112] R. Shepard, Hamiltonian Matrix and Reduced Density Matrix Construction with Nonlinear Wave Functions. J. Phys. Chem. A 110, (2006), 8880. 
[113] R. Shepard, M. Minkoff, Optimization of Nonlinear Wave Function Parameters. Int. J. Quantum Chem. 106, (2006), 3190.

[114] S. R. Brozell, R. Shepard, Z. Zhang, Spin-orbit Interaction with Nonlinear Wave Functions. Int. J. Quantum Chem. 107, (2007), 3191.

[115] R. Shepard, M. Minkoff, S. R. Brozell, Nonlinear Wave Function Expansions: A Progress Report. Int. J. Quantum Chem. 107, (2007), 3203.

[116] R. Shepard, G. Gidofalvi, S. R. Brozell, The Multifacet Graphically Contracted Function Method. I. Formulation and Implementation. J. Chem. Phys. 141, (2014), 064105.

[117] S. Sharma, A. Alavi, Multireference Linearized Coupled Cluster Theory for Strongly Correlated Systems using Matrix Product States. J. Chem. Phys. 143, (2015), 102815.

[118] J. J. Lutz, P. Piecuch, Performance of the Completely Renormalized Equation-of-motion Coupled-cluster Method in Calculations of Excited-state Potential Cuts of Water. Comp. Theor. Chem. 1040-1041, (2014), 20.

[119] T. Van Voorhis, M. Head-Gordon, Implementation of Generalized Valence Bond-inspired Coupled Cluster Theories. J. Chem. Phys. 117, (2002), 9190.

[120] T. H. Henderson, I. W. Bulik, T. Stein, G. E. Scuseria, Seniority-based Coupled Cluster Theory. J. Chem. Phys. 141, (2014), 244104.

[121] T. H. Henderson, I. W. Bulik, G. E. Scuseria, Pair Extended Coupled Cluster Doubles. J. Chem. Phys. 142, (2015), 214116.

[122] A. I. Krylov, Size-consistent Wave Functions for Bond-breaking: the Equation-of-motion Spin-flip Model. Chem. Phys. Lett. 338, (2001), 375. 
[123] A. D. Chien, P. M. Zimmerman, Recovering Dynamic Correlation in Spin Flip Configuration Interaction through a Difference Dedicated Approach.

J. Chem. Phys. 146, (2017), 014103.

[124] C. A. Jiménez-Hoyos, T. M. Henderson, T. Tsuchimochi, G. E. Scuseria, Projected Hartree-Fock Theory. J. Chem. Phys. 136, (2012), 164109.

[125] L. Bytautas, C. A. Jiménez-Hoyos, R. Rodríguez-Guzmán, G. E. Scuseria, Potential Energy Curves for Mo2: Multi-component Symmetry-projected Hartree-Fock and Beyond. Mol. Phys. 112, (2014), 1938.

[126] J. M. Wahlen-Strothman, T. M. Henderson, M. R. Hermes, M. Degroote, Y. Qiu, J. Zhao, J. Dukelsky, G. E. Scuseria, Merging Symmetry Projection Methods with Coupled Cluster Theory: Lessons from the Lipkin Model Hamiltonian. J. Chem. Phys. 146, (2017), 054110.

[127] S. Lehtola, J. Parkhill, M. Head-Gordon, Cost-effective Description of Strong Correlation: Efficient Implementations of the Perfect Quadruples and Perfect Hextuples Models. J. Chem. Phys. 145, (2016), 134110.

[128] G. H. Booth, D. Cleland, A. J. W. Thom, A. Alavi, Breaking the Carbon Dimer: the Challenges of Multiple Bond Dissociation with Full Configuration Interaction Quantum Monte Carlo Methods. J. Chem. Phys. 135, (2011), 084104.

[129] G. H. Booth, S. D. Smart, A. Alavi, Linear-scaling and Parallelisable Algorithms for Stochastic Quantum Chemistry. Mol. Phys. 112, (2014), 1855. 
[130] M. A. Morales, J. McMinis, B. K. Clark, J. Kim, G. E. Scuseria, Multideterminant Wave Functions in Quantum Monte Carlo. J. Chem. Theory Comput. 8, (2012), 2181.

[131] M. C. Per, D. M. Cleland, Energy-based Truncation of Multi-determinant Wavefunctions in Quantum Monte Carlo. J. Chem. Phys. 146, (2017), 164101.

[132] K. Boguslawski, P. Tecmer, G. Barcza, Ö. Legeza, M. Reiher,

Orbital Entanglement in Bond-Formation Processes. J. Chem. Theory Comput. 9, (2013), 2959.

[133] G. Racah, Theory of Complex Spectra. IV.

Phys. Rev. 76, (1949), 1352.

[134] A. K. Kerman, Pairing Forces and Nuclear Collective Motion. Ann. Phys. (NY) 12, (1961), 300.

[135] I. Talmi, Simple Models of Complex Nuclei, Harwood Academic Publishers, (1993).

[136] K. Boguslawski, P. Tecmer, P. W. Ayers, P. Bultinck, S. De Beerdemacher, D. Van Neck, Efficient Description of Strongly Correlated Electrons with Mean-field Cost. Phys. Rev. B 89, (2014), 201106 (R).

[137] Z. Chen, C. Zhou, W. Wu, Seniority Number in Valence Bond Theory. J. Chem. Theory Comput. 11, (2015), 4102.

[138] R. Gebauer, M. H. Cohen, R. Car, A Well-scaling Natural Orbital Theory. Proc. Nat. Academy of Sci. USA 113, (2016), 12913.

[139] J. J. Shepherd, T. M. Henderson, G. E. Scuseria, Using Full Configuration Interaction Quantum Monte Carlo in a Seniority Zero Space to Investigate the Correlation Energy Equivalence of Pair Coupled Cluster Doubles . J. Chem. Phys. 144, (2016), 094112. 
[140] P. A. Limacher, A New Wavefunction Hierarchy for Interacting Geminals. J. Chem. Phys. 145, (2016), 194102.

[141] K. G. Wilson, The Renormalization Group: Critical Phenomena and the Kondo Problem. Rev. Mod. Phys. 47, (1975), 773.

[142] K. G. Wilson, The Renormalization Group and Critical Phenomena. Rev. Mod. Phys. 55, (1983), 583.

[143] K. H. Marti, M. Reiher, The Density Matrix Renormalization Group Algorithm in Quantum Chemistry. Z. Phys. Chem. 224, (2010), 583.

[144] W. Hofstetter, Generalized Numerical Renormalization Group for Dynamical Quantities. Phys. Rev. Lett. 85, (2000), 1508.

[145] M. W. Schmidt, K. K. Baldridge, J. A. Boatz, S. T. Elbert, M. S. Gordon, J. H. Jensen, S. Koseki, N. Matsunaga, K. A. Nguyen, S. J. Su, T. L. Windus, M. Dupuis, J. A. Montgomery, General Atomic and Molecular Electronic Structure System. J. Comput. Chem. 14, (1993), 1347.

[146] E. R. Davidson, Properties and Uses of Natural Orbitals. Rev. Mod. Phys. 44, (1972), 451.

[147] W. C. Lu, C. Z. Wang, M. W. Schmidt, L. Bytautas, K. M. Ho, K. Ruedenberg, Molecule Intrinsic Minimal Basis Sets. I. Exact Resolution of ab initio Optimized Molecular Orbitals in Terms of Deformed Atomic Minimal-basis Orbitals.

J. Chem. Phys. 120, (2004), 2629.

[148] W. C. Lu, C. Z. Wang, M. W. Schmidt, L. Bytautas, K. M. Ho, K. Ruedenberg, Molecule Intrinsic Minimal Basis Sets. II. Bonding Analyses for $\mathrm{Si}_{4} \mathrm{H}_{6}$ and $\mathrm{Si}_{2}$ to $\mathrm{Si}_{10}$. J. Chem. Phys. 120, (2004), 2638. 
[149] E. Neuscamman, Communication: A Jastrow Factor Coupled Cluster Theory for Weak and Strong Electron Correlation. J. Chem. Phys. 139, (2013), 181101.

[150] L. Zhao, E. Neuscamman, Amplitude Determinant Coupled Cluster with Pairwise Doubles. J. Chem. Theory Comput. 12, (2016), 5841.

[151] T. Tsuchimochi, S. Ten-no, Black-Box Description of Electron Correlation with the Spin-Extended Configuration Interaction Model: Implementation and Assessment. J. Chem. Theory Comput. 12, (2016), 1741.

[152] J. Hachmann, W. Cardoen, G. K.-L. Chan, Multireference Correlation in Long Molecules with the Quadratic Scaling Density Matrix Renormalization Group. J. Chem. Phys. 125, (2006), 144101.

[153] T. Tsuchimochi, G. E. Scuseria, Strong Correlations via Constrained-pairing Mean-field Theory. J. Chem. Phys. 131, (2009), 121102.

[154] A. V. Sinitskiy, L. Greenman, D. A. Mazziotti, Strong Correlation in Hydrogen Chains and Lattices using the Variational Two-electron Reduced Density Matrix Method. J. Chem. Phys. 133, (2010), 014104.

[155] L. Stella, C. Attaccalite, S. Sorella, A. Rubio, Strong Electronic Correlation in the Hydrogen Chain: A Variational Monte Carlo Study. Phys. Rev. B 84, (2011), 245117.

[156] M. Motta, D. M. Ceperly, G. K.-L. Chan, J. A. Gomez, E. Gull, et. al. Towards the Solution of the Many-electron Problem in Real Materials: Equation of State of the Hydrogen Chain with State-of-the-art Many-body Methods. Phys. Rev. X 7, (2017), 031059. 
[157] W. Klopper, Limiting Values for Møller-Plesset Second-order Correlation Energies of Polyatomic Systems: A Benchmark Study on $\mathrm{Ne}, \mathrm{HF}, \mathrm{H}_{2} \mathrm{O}, \mathrm{N}_{2}$, and $\mathrm{He} . . . \mathrm{He}$. J. Chem. Phys. 102, (1995), 6168. 
Table 1. Seniority number $(\Omega)$ sectors in terms of numbers of Slater determinants in wave functions considered in the present study.

$\Omega$-sectors $\quad \begin{gathered}\text { Number of Slater } \\ \text { Determinants }\end{gathered} \quad K$-value ${ }^{\text {a) }} \quad$ Maximum Matrix-Dimension

I. $\mathrm{N}_{2}$ : 2-core-orbitals, Full-Valence-Space $=(10$ electrons in 8 orbitals $), \mathbf{A g}_{\mathrm{g}-S y m m e t r y}$

$\begin{array}{lrrr}\Omega=0 & 56 & 1 & \\ \Omega=2 & 60 & 2 & \\ \Omega=4 & 240 & 6 & \\ \Omega=6 & 40 & 20 & \\ \Omega=\mathbf{0 , 2 , 4 , 6} & \mathbf{3 9 6} & & \mathbf{3 9 6} \times \mathbf{3 9 6} \\ \text { FCI } & & & \mathbf{2 8} \times \mathbf{2 8} \\ \boldsymbol{\Omega} \text {-ERG }(\boldsymbol{m}, \boldsymbol{M}) & & & \end{array}$

II. Hs: Full-Valence-Space $=(8$ electrons in 8 orbitals $)$, Ag-Symmetry

$\begin{array}{lrrr}\Omega=0 & 70 & 1 \\ \Omega=2 & 480 & 2 & \\ \Omega=4 & 1368 & 6 & \\ \Omega=6 & 480 & 20 & \\ \Omega=8 & 70 & 70 & \\ \Omega=\mathbf{0 , 2 , 4 , 6 , 8} & \mathbf{2 4 6 8} & & \mathbf{2 4 6 8} \times \mathbf{2 4 6 8} \\ \text { FCI } & & & \mathbf{9 0} \times \mathbf{9 0} \text { b) } \\ \Omega \text {-ERG }(\boldsymbol{m}, \boldsymbol{M}) & & & \end{array}$

III. $\mathrm{H}_{2} \mathrm{O}$ : 1-core-orbital, Full-Valence-Space $=(8$ electrons in 12 orbitals $), \mathrm{A}_{1-\mathrm{Symmetry}}$

$\Omega=0$

$\Omega=2$

$\Omega=4$

$\Omega=6$

$\Omega=8$

$\Omega=0,2,4,6,8$

FCI matrix: $\Omega-\operatorname{ERG}(m, M)$

$\begin{array}{rr}495 & 1 \\ 5280 & 2 \\ 21336 & 6 \\ 25440 & 20 \\ 8890 & 70 \\ \mathbf{6 1 4 4 1} & \end{array}$

1

2

6

20

70

a) The $K$-value used in the $\Omega$-ERG $(m, M)$ procedure (see text) for each $\Omega$-sector.

b) Largest matrix diagonalized in the $\Omega-\operatorname{ERG}(m, M)$ procedure. 
Table 1 (continue). Seniority number $(\Omega)$ sectors in terms of numbers of Slater determinants in wave functions considered in the present study.

$\Omega$-sectors

Number of Slater $K$-value ${ }^{\text {a) }}$

Maximum Matrix-Dimension

Determinants

IV. C2: 2-core-orbitals, Full-Valence-Space $=(8$ electrons in 16 orbitals $), A_{1 g}-$ symmetry

$\Omega=0$

$\Omega=2$

$\Omega=4$

$\Omega=6$

$\Omega=8$

$\Omega=0,2,4,6,8$

FCI

$\Omega-\operatorname{ERG}(m, M)$

$\begin{array}{rr}1,820 & 1 \\ 11,648 & 2 \\ 93,456 & 6 \\ 188,800 & 20 \\ 119,140 & 70\end{array}$

414,864

$84 \times 84^{\text {b) }}$

a) The $K$-value used in the $\Omega$-ERG $(m, M)$ procedure (see text) for each $\Omega$-sector.

b) Largest matrix diagonalized in the $\Omega-\operatorname{ERG}(m, M)$ procedure. 
Table 2. $\mathrm{N}_{2}$ (STO-3G basis set). The ordering of the determinants in terms of their electron occupations in valence molecular orbitals for $\Omega=0$ sector ( 56 determinants). For Full valence space, FCI $=$ CAS $[10,8]$. Core $(1 \mathrm{~s})$ orbitals on Nitrogen atoms are kept doubly occupied and are not shown.

No $\quad$ Molecular Orbital electron occupations a)

$$
\begin{array}{llllllll}
\mathbf{A g}_{\mathrm{g}} & \mathbf{B}_{1 \mathrm{u}} & \mathbf{A}_{\mathrm{g}} & \mathbf{B}_{3 \mathrm{u}} & \mathbf{B}_{2 \mathrm{u}} & \mathbf{B}_{3 \mathrm{~g}} & \mathbf{B}_{2 \mathrm{~g}} & \mathbf{B}_{1 \mathrm{u}}
\end{array}
$$

\begin{tabular}{lllllllll}
\hline $\mathbf{1}$ & 2 & 2 & 2 & 2 & 2 & - & - & - \\
$\mathbf{2}$ & 2 & 2 & 2 & 2 & - & 2 & - & - \\
$\mathbf{3}$ & 2 & 2 & 2 & 2 & - & - & 2 & - \\
$\mathbf{4}$ & 2 & 2 & 2 & 2 & - & - & - & 2 \\
$\mathbf{5}$ & 2 & 2 & 2 & - & 2 & 2 & - & - \\
$\mathbf{6}$ & 2 & 2 & 2 & - & 2 & - & 2 & - \\
$\mathbf{7}$ & 2 & 2 & 2 & - & 2 & - & - & 2 \\
$\mathbf{8}$ & 2 & 2 & 2 & - & - & 2 & 2 & - \\
$\mathbf{9}$ & 2 & 2 & 2 & - & - & 2 & - & 2 \\
$\mathbf{1 0}$ & 2 & 2 & 2 & - & - & - & 2 & 2 \\
$\mathbf{1 1}$ & 2 & 2 & - & 2 & 2 & 2 & - & - \\
$\mathbf{1 2}$ & 2 & 2 & - & 2 & 2 & - & 2 & - \\
$\mathbf{1 3}$ & 2 & 2 & - & 2 & 2 & - & - & 2 \\
$\mathbf{1 4}$ & 2 & 2 & - & 2 & - & 2 & 2 & - \\
$\mathbf{1 5}$ & 2 & 2 & - & 2 & - & 2 & - & 2 \\
$\mathbf{1 6}$ & 2 & 2 & - & 2 & - & - & 2 & 2 \\
$\mathbf{1 7}$ & 2 & 2 & - & - & 2 & 2 & 2 & - \\
$\mathbf{1 8}$ & 2 & 2 & - & - & 2 & 2 & - & 2 \\
$\mathbf{1 9}$ & 2 & 2 & - & - & 2 & - & 2 & 2 \\
$\mathbf{2 0}$ & 2 & 2 & - & - & - & 2 & 2 & 2 \\
$\mathbf{2 1}$ & 2 & - & 2 & 2 & 2 & 2 & - & - \\
$\mathbf{2 2}$ & 2 & - & 2 & 2 & 2 & - & 2 & - \\
$\mathbf{2 3}$ & 2 & - & 2 & 2 & 2 & - & - & 2 \\
$\mathbf{2 4}$ & 2 & - & 2 & 2 & - & 2 & 2 & - \\
$\mathbf{2 5}$ & 2 & - & 2 & 2 & - & 2 & - & 2 \\
$\mathbf{2 6}$ & 2 & - & 2 & 2 & - & - & 2 & 2 \\
$\mathbf{2 7}$ & 2 & - & 2 & - & 2 & 2 & 2 & - \\
$\mathbf{2 8}$ & 2 & - & 2 & - & 2 & 2 & - & 2 \\
$\mathbf{2 9}$ & 2 & - & 2 & - & 2 & - & 2 & 2 \\
& & & & & & & &
\end{tabular}

a) Molecular Orbital Symmetry-labels of $\mathrm{D}_{2 \mathrm{~h}}$ point group (irreducible representation labels) are listed below. 
Table 2 (continue). $\mathrm{N}_{2}$ (STO-3G basis set). The ordering of the determinants in terms of their electron occupations in valence molecular orbitals for $\Omega=0$ sector (56 determinants). For Full valence space, FCI $=$ CAS $[10,8]$. Core $(1 \mathrm{~s})$ orbitals on Nitrogen atoms are kept doubly occupied and are not shown.

No $\quad$ Molecular Orbital electron occupations ${ }^{\text {a) }}$
Ag
$\mathbf{B}_{1 \mathbf{u}}$
$\mathbf{A g}$
B3u
$\mathbf{B}_{2 \mathbf{u}}$
B3g

$\mathbf{B}_{2 \mathrm{~g}} \quad \mathbf{B}_{1 \mathrm{u}}$

30

31

32

33

34

35

36

37

38

39

40

41

42

43

44

45

46

47

48

49

50

51

52

53

54

55

56

$\begin{array}{llllllll}2 & - & 2 & - & - & 2 & 2 & 2 \\ 2 & - & - & 2 & 2 & 2 & 2 & - \\ 2 & - & - & 2 & 2 & 2 & - & 2 \\ 2 & - & - & 2 & 2 & - & 2 & 2 \\ 2 & - & - & 2 & - & 2 & 2 & 2 \\ 2 & - & - & - & 2 & 2 & 2 & 2 \\ - & 2 & 2 & 2 & 2 & 2 & - & - \\ - & 2 & 2 & 2 & 2 & - & 2 & - \\ - & 2 & 2 & 2 & 2 & - & - & 2 \\ - & 2 & 2 & 2 & - & 2 & 2 & - \\ - & 2 & 2 & 2 & - & 2 & - & 2 \\ - & 2 & 2 & 2 & - & - & 2 & 2 \\ - & 2 & 2 & - & 2 & 2 & 2 & - \\ - & 2 & 2 & - & 2 & 2 & - & 2 \\ - & 2 & 2 & - & 2 & - & 2 & 2 \\ - & 2 & 2 & - & - & 2 & 2 & 2 \\ - & 2 & - & 2 & 2 & 2 & 2 & - \\ - & 2 & - & 2 & 2 & 2 & - & 2 \\ - & 2 & - & 2 & 2 & - & 2 & 2 \\ - & 2 & - & 2 & - & 2 & 2 & 2 \\ - & 2 & - & - & 2 & 2 & 2 & 2 \\ - & - & 2 & 2 & 2 & 2 & 2 & - \\ - & - & 2 & 2 & 2 & 2 & - & 2 \\ - & - & 2 & 2 & 2 & - & 2 & 2 \\ - & - & 2 & 2 & - & 2 & 2 & 2 \\ - & - & 2 & - & 2 & 2 & 2 & 2 \\ - & - & - & 2 & 2 & 2 & 2 & 2\end{array}$

a) Molecular Orbital Symmetry-labels of $\mathrm{D}_{2 \mathrm{~h}}$ point group (irreducible representation labels) are listed below. 
Table 3. Deviations of $\Omega$-ERG $(m=4, M=4)$ energies (in hartree) from FCI energies for lowest-energy states ${ }^{1)}$ for $\mathrm{N}_{2}(\mathrm{STO}-3 \mathrm{G}$ basis set, $\mathrm{R}=2.4 \AA$ ). The spatial-symmetry is $\mathrm{A}_{\mathrm{g}}$, point group used is $\mathrm{D}_{2 \mathrm{~h}}$.

Number of RG Sweeps

\begin{tabular}{cllll}
\hline 2 & 0.0051652818 & 0.0044496662 & 0.1456535941 & 0.1590419544 \\
3 & 0.0013537475 & 0.0009110845 & 0.1390419294 & 0.1583211429 \\
4 & 0.0005461460 & 0.0001658260 & 0.1336763961 & 0.1579593797 \\
5 & 0.0002266860 & 0.0000292291 & 0.1290447494 & 0.1574514368 \\
6 & 0.0000849822 & 0.0000051067 & 0.1239403801 & 0.1533874528 \\
7 & 0.0000281037 & 0.0000008789 & 0.1020435056 & 0.1146425219 \\
8 & 0.0000082270 & 0.0000001576 & 0.0461757686 & 0.0592423566 \\
9 & 0.0000021474 & 0.0000000371 & 0.0196591882 & 0.0253456285 \\
10 & 0.0000005091 & 0.0000000119 & 0.0102294317 & 0.0124252680 \\
11 & 0.0000001187 & 0.0000000041 & 0.0057474957 & 0.0083262675 \\
12 & 0.0000000338 & 0.0000000014 & 0.0032838167 & 0.0067023590 \\
14 & 0.0000000069 & 0.0000000001 & 0.0010890944 & 0.0049232101 \\
16 & 0.0000000015 & 0.0 & 0.0003529161 & 0.0036175490 \\
18 & 0.0000000002 & 0.0 & 0.0001118301 & 0.0025835821 \\
20 & 0.0 & 0.0 & 0.0000355839 & 0.0017967932 \\
22 & 0.0 & 0.0 & 0.0000118594 & 0.0012237353 \\
24 & 0.0 & 0.0 & 0.0000043698 & 0.0008207318 \\
26 & 0.0 & 0.0 & 0.0000018604 & 0.0005445239 \\
28 & 0.0 & 0.0 & 0.0000009170 & 0.0003586064 \\
30 & 0.0 & 0.0 & 0.0000005034 & 0.0002349956 \\
32 & 0.0 & 0.0 & 0.0000002938 & 0.0001534858 \\
48 & 0.0 & 0.0 & 0.0000000056 & 0.0000048899 \\
\hline 10 & & & & \\
\hline
\end{tabular}

1) $-107.4412958770 \mathrm{~h}\left({ }^{1} \mathrm{~A}_{\mathrm{g}}\right) ;-107.4350980802 \mathrm{~h}\left({ }^{5} \mathrm{~A}_{\mathrm{g}}\right) ;-107.3211561796 \mathrm{~h}\left({ }^{5} \mathrm{~A}_{\mathrm{g}}\right) ;-107.3189648501 \mathrm{~h}\left({ }^{3} \mathrm{~A}_{\mathrm{g}}\right)$; 
Table 4. Deviations of $\Omega$-ERG $(m=4, M=8)$ energies (in hartree) from FCI energies for lowest-energy states ${ }^{1)}$ for $\mathrm{N}_{2}(\mathrm{STO}-3 \mathrm{G}$ basis set, $R=2.4 \AA$ ). The spatial-symmetry is $A_{g}$, point group used is $D_{2 h}$.

\begin{tabular}{|c|c|c|c|c|c|}
\hline $\begin{array}{l}\text { Number of } \\
\text { RG Sweeps }\end{array}$ & $\begin{array}{l}f \\
s\end{array}$ & 2 & 3 & 4 & 5 \\
\hline 2 & 0.0043967655 & 0.0044496659 & 0.0023126751 & 0.1443790153 & 0.1447959052 \\
\hline 3 & 0.0006285494 & 0.0009110842 & 0.0021995496 & 0.1375120631 & 0.1377730945 \\
\hline 4 & 0.0001297971 & 0.0001658189 & 0.0021932437 & 0.1319704433 & 0.1310346840 \\
\hline 5 & 0.0000315033 & 0.0000289855 & 0.0021924321 & 0.1270893953 & 0.1249041311 \\
\hline 6 & 0.0000077773 & 0.0000042939 & 0.0021921720 & 0.0619839053 & 0.1090835815 \\
\hline 7 & 0.0000018780 & 0.0000005661 & 0.0021920148 & 0.0134780129 & 0.1057057533 \\
\hline 8 & 0.0000004440 & 0.0000000604 & 0.0021918955 & 0.0012312488 & 0.0609172972 \\
\hline 9 & 0.0000001012 & 0.0000000074 & 0.0007574603 & 0.0000004696 & 0.0216578169 \\
\hline 10 & 0.0000000217 & 0.0000000009 & 0.0001767301 & 0.0000003901 & 0.0064126635 \\
\hline 11 & 0.0000000043 & 0.0000000001 & 0.0000425188 & 0.0000003242 & 0.0018273730 \\
\hline 12 & 0.0000000008 & 0.0 & 0.0000105175 & 0.0000002688 & 0.0005123274 \\
\hline 16 & 0.0 & 0.0 & 0.0000000538 & 0.0000000974 & 0.0000031019 \\
\hline 24 & 0.0 & 0.0 & 0.0000000001 & 0.0000000013 & 0.0000000401 \\
\hline 32 & 0.0 & 0.0 & 0.0 & 0.0 & 0.0000000004 \\
\hline
\end{tabular}

1) $-107.4412958770 \mathrm{~h}\left({ }^{1} \mathrm{~A}_{\mathrm{g}}\right) ;-107.4350980802 \mathrm{~h}\left({ }^{5} \mathrm{~A}_{\mathrm{g}}\right) ;-107.3211561796 \mathrm{~h}\left({ }^{5} \mathrm{~A}_{\mathrm{g}}\right) ;-107.3189648501 \mathrm{~h}\left({ }^{3} \mathrm{~A}_{\mathrm{g}}\right) ;-107.3048555744 \mathrm{~h}\left({ }^{5} \mathrm{~A}_{\mathrm{g}}\right)$. 
Table 5. $\mathrm{H}_{8}$ (STO-3G basis set, $\mathrm{A}_{\mathrm{g}}$-electronic states). MOs are ordered according to their orbital energies. $\mathrm{R}=1.8 \mathrm{Angstrom}$.

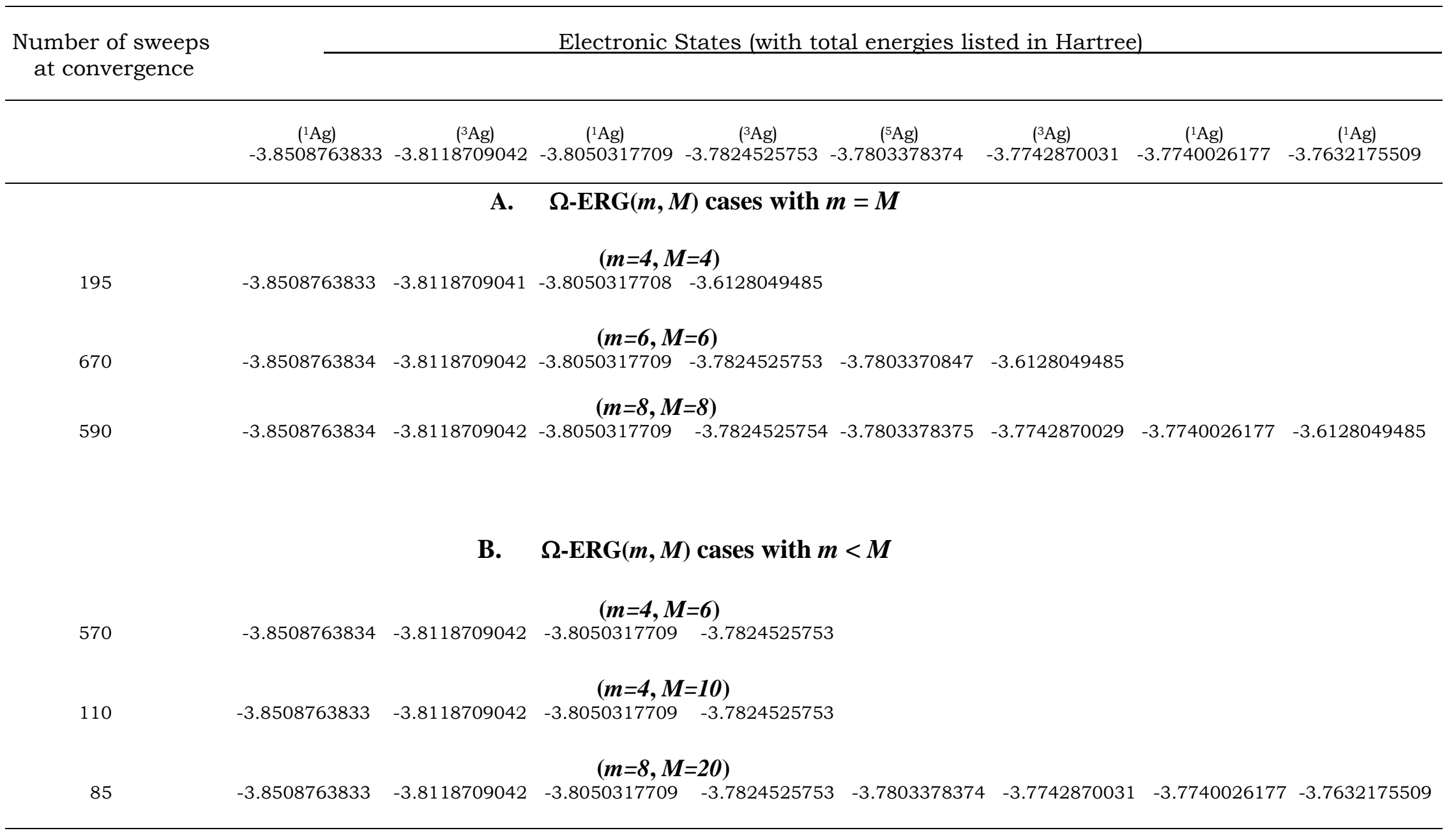


Table 6. The convergence of total energies towards FCI benchmarks for lowest states for $\mathrm{C}_{2}$ (6-31G basis set, $\mathrm{R}=1.2 \AA$ / near-equilibrium bond-length) with respect to numbers of RGsweeps or CI-iterations. The spatial-symmetry is $\mathrm{A}_{\mathrm{g}}$, point group used is $\mathrm{D}_{2 \mathrm{~h}}$. The number of FCI determinants is 414,864. Listed are deviations (in hartree) from the benchmark energies.

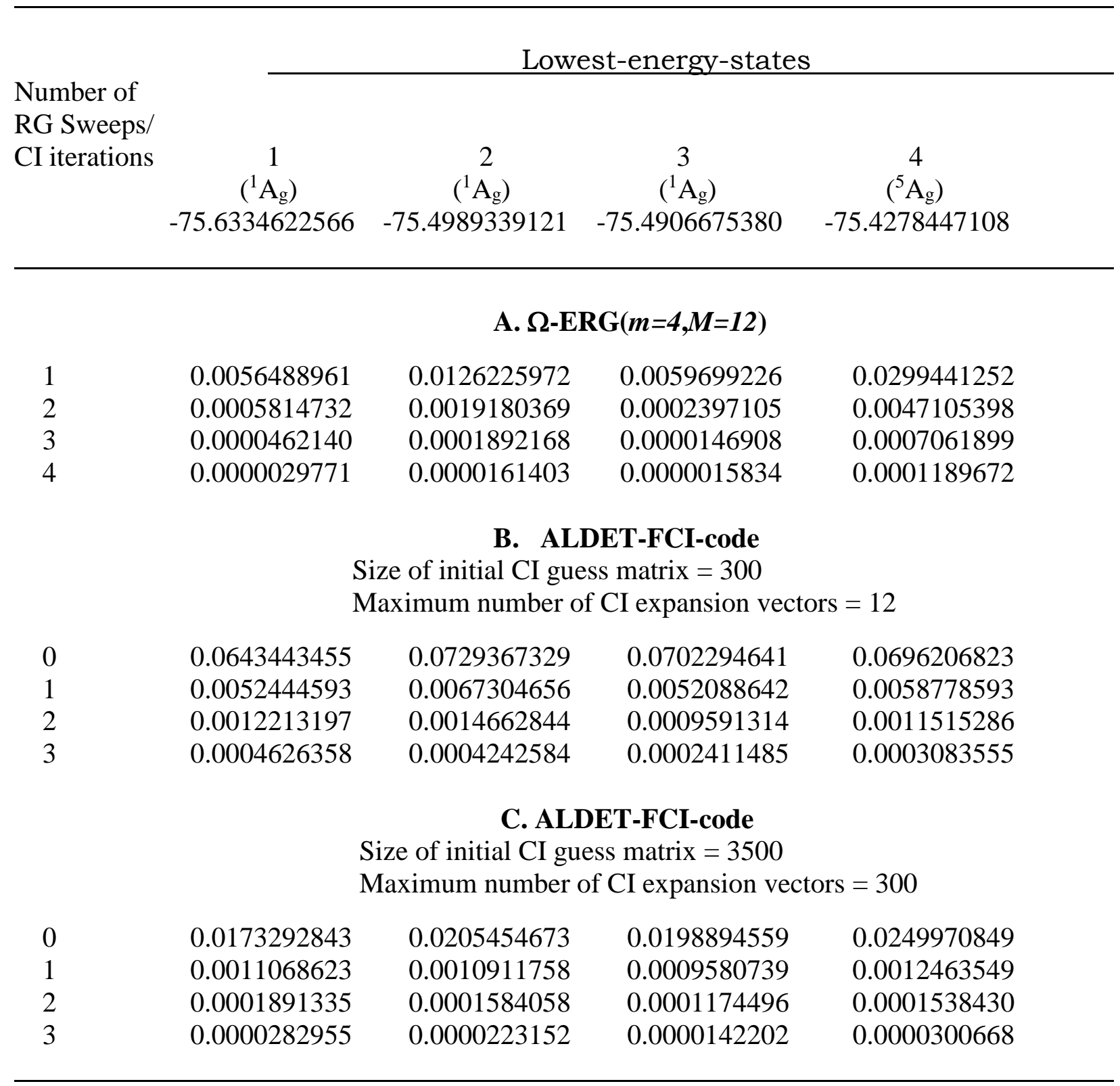


Table 7. The convergence of total energies towards FCI benchmarks for lowest states for $\mathrm{C}_{2}$ (6-31G basis set, $\mathrm{R}=1.8 \AA$ ) with respect to numbers of RG-sweeps or CI-iterations. The spatialsymmetry is $\mathrm{Ag}_{\mathrm{g}}$, point group used is $\mathrm{D}_{2 \mathrm{~h}}$. The number of FCI determinants is 414,864 . Listed are deviations (in hartree) from the benchmark energies.

Lowest-energy-states

Number of

RG Sweeps

$\begin{array}{ccc}1 & 2 & 3 \\ \left({ }^{1} \mathrm{~A}_{\mathrm{g}}\right) & \left({ }^{1} \mathrm{~A}_{\mathrm{g}}\right. & \left({ }^{\mathrm{A}}\right) \\ -75.5158646401 & -75.5150157586 & -75.5056588286\end{array}$

\section{A. $\Omega$-ERG $(m=3, M=10)$}

1

2

3

4

5

6

0.0103983800

0.0139173727

0.0155096453

0.0015540163

0.0025423520

0.0037793127

0.0009080528

0.0001146815

0.0012007760

0.0003059512

0.0000086333

0.0004486052

0.0001002295

0.0000011759

0.0001707232

0.0000318861

0.0000001624

0.0000633700

B. $\Omega$-ERG $(m=3, M=14)$

$\begin{array}{llll}1 & 0.0105348634 & 0.0141697302 & 0.0157741622 \\ 2 & 0.0015706462 & 0.0022649604 & 0.0038283200 \\ 3 & 0.0007886201 & 0.0000611969 & 0.0012101892 \\ 4 & 0.0002402964 & 0.0000067008 & 0.0004508604 \\ 5 & 0.0000782504 & 0.0000008776 & 0.0001694785\end{array}$




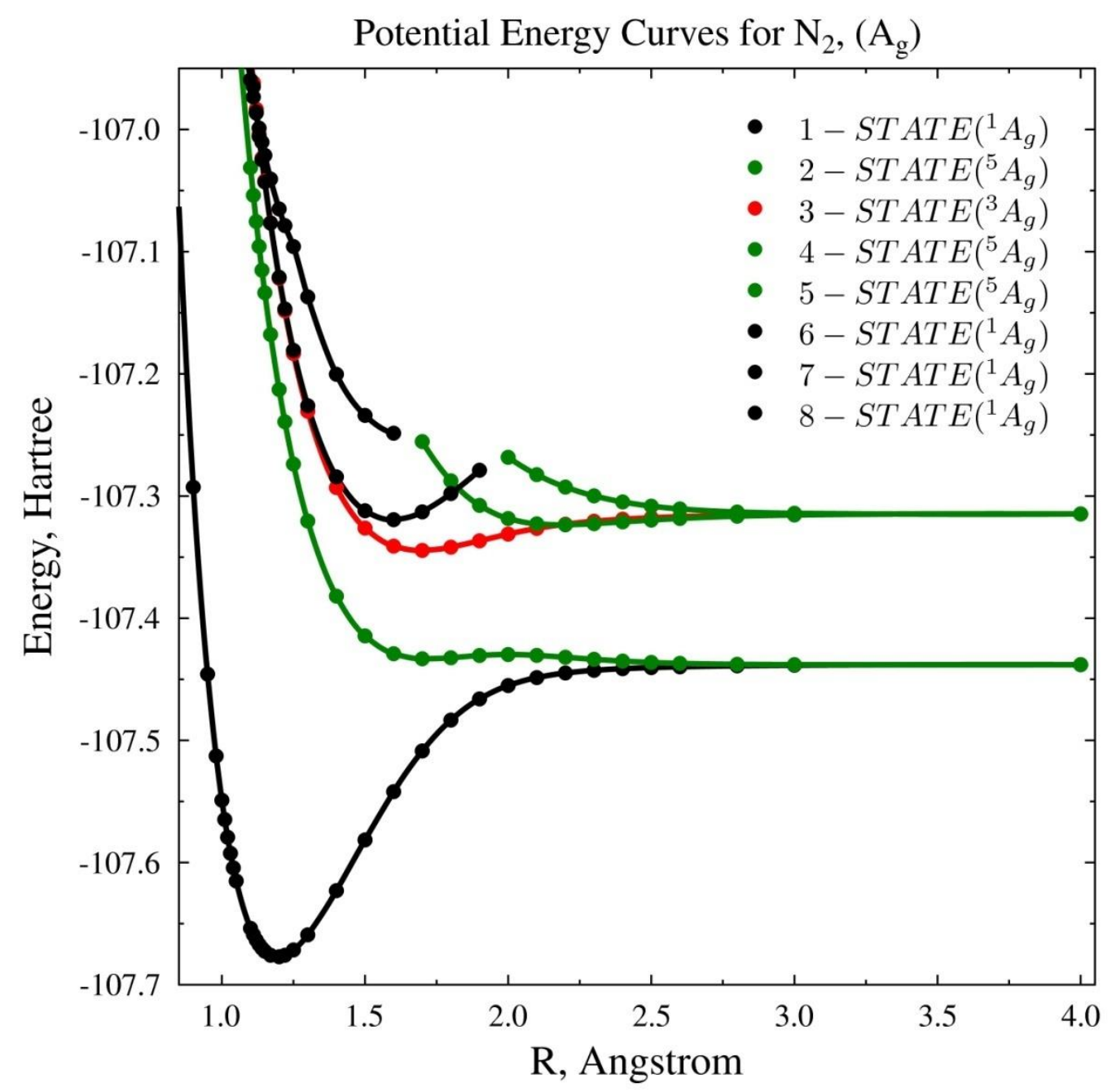

Figure 1. $\mathrm{N}_{2}$ (STO-3G basis set). Only five lowest states $\left(\mathrm{A}_{\mathrm{g}}\right)$ potential energy curves at FCI level of theory at each $\mathrm{R}$ value are displayed. 


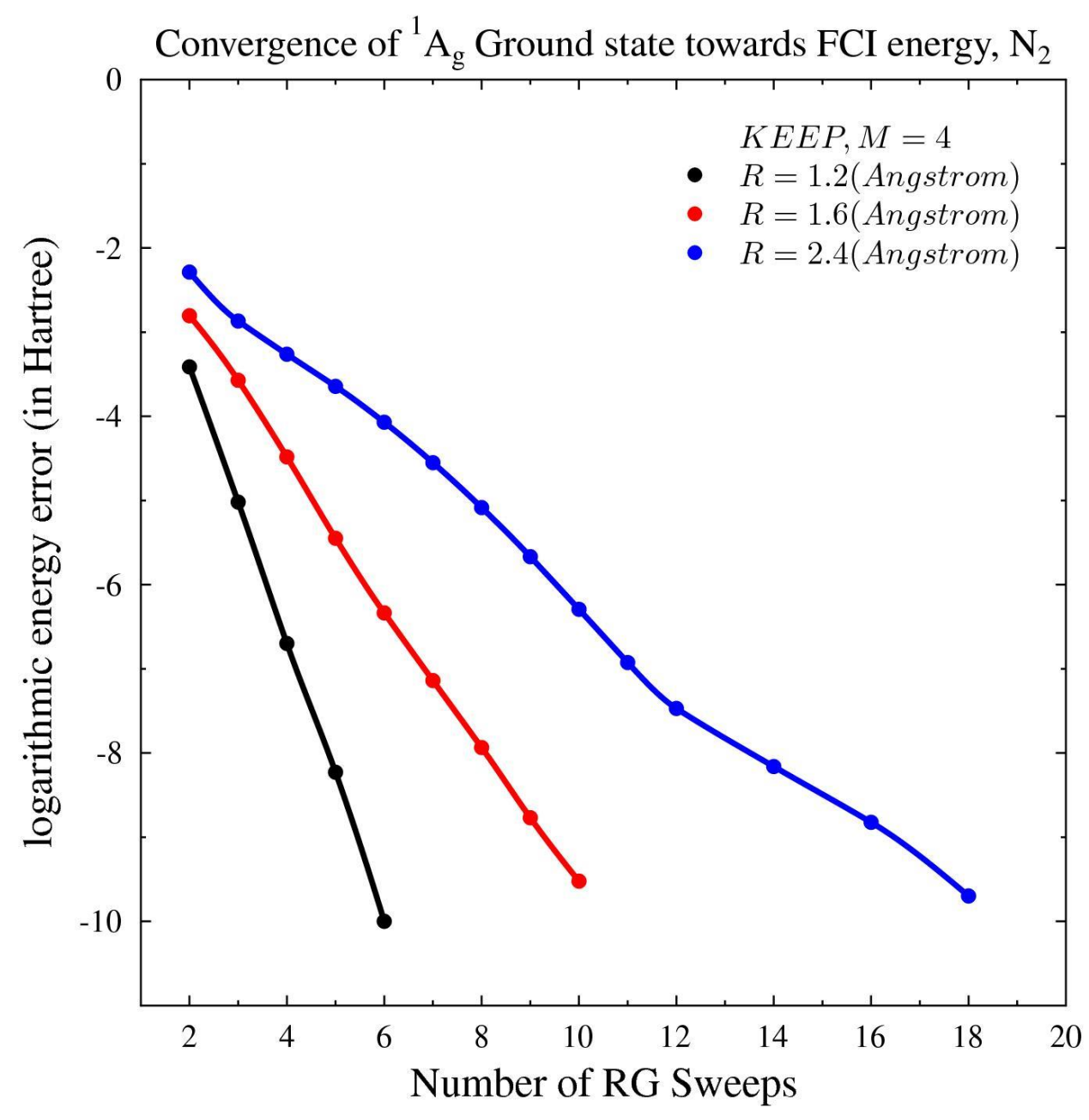

Figure 2. $\mathrm{N}_{2}(\mathrm{STO}-3 \mathrm{G})$. Graphical display of the $\Omega$-ERG $(m=1, M=4)$ convergence rates for the ground-state energy: logarithmic energy error versus the number of $R G$ sweeps for internuclear distances $R=1.2 \AA(E=-107.6770854427 \mathrm{~h})$, $1.6 \AA(\mathrm{E}=-107.5419619206 \mathrm{~h})$ and $2.4 \AA(\mathrm{E}=-107.4412958770 \mathrm{~h})$. 
Convergence of $\mathrm{A}_{\mathrm{g}}$ states towards $\mathrm{FCI}$ energies, $\mathrm{N}_{2}$ at $\mathrm{R}=2.4 \mathrm{~A}$

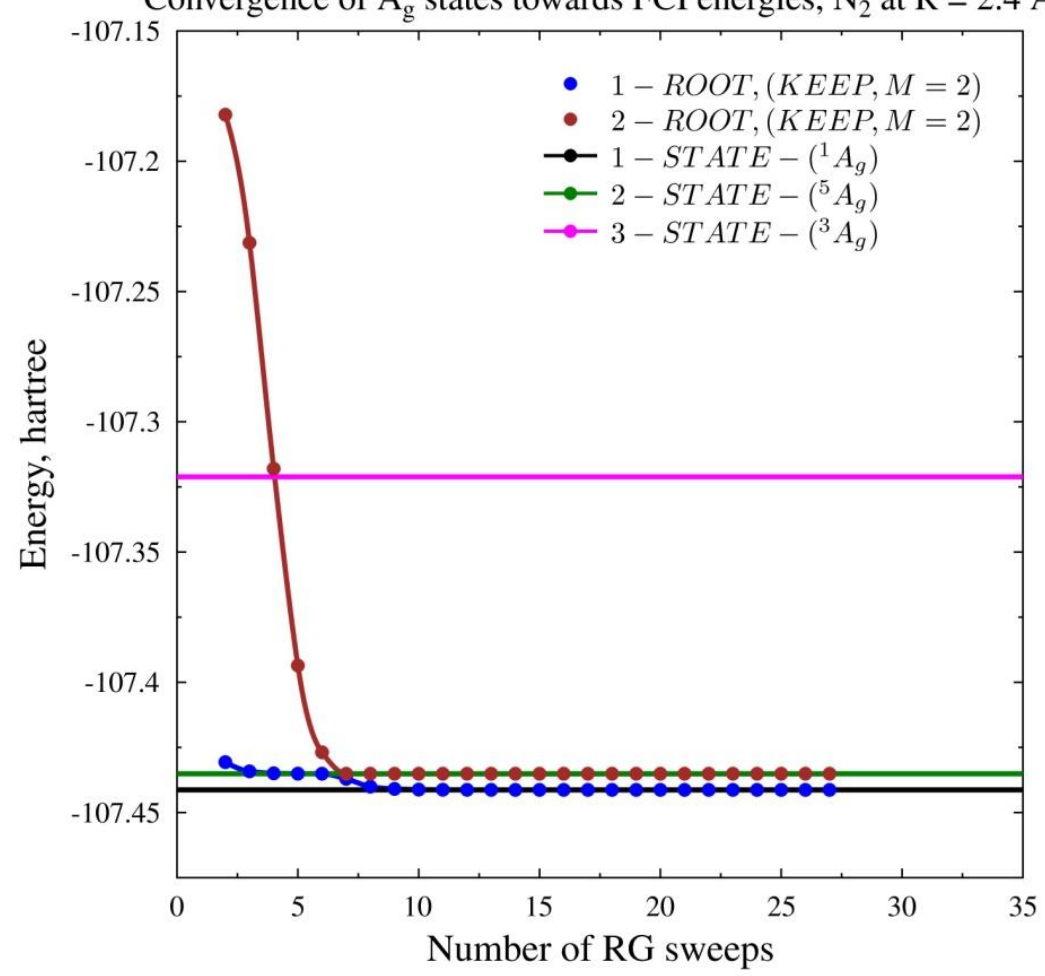

Convergence of $\mathrm{A}_{\mathrm{g}}$ states towards FCI energies, $\mathrm{N}_{2}$ at $\mathrm{R}=2.4 \mathrm{~A}$

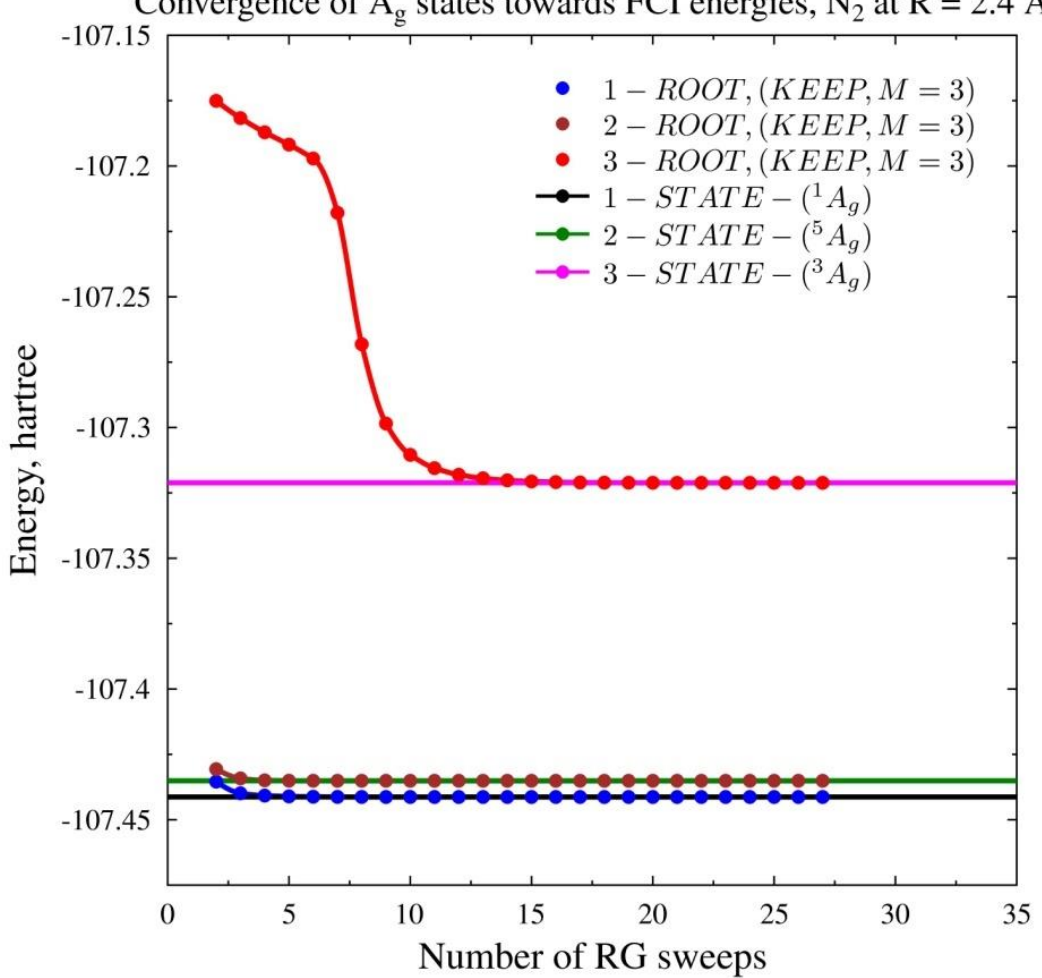

Figure 3. $\mathrm{N}_{2}$ (STO-3G, $\mathrm{R}=2.4 \AA$ ). Comparison of total energy convergence rates $\left(\mathrm{A}_{\mathrm{g}}\right.$-states) for two cases: (Left-panel) $\Omega$ $\operatorname{ERG}(m=2, M=2)$ when two lowest-energy states are kept after each iteration/sweep; (Right-panel) $\Omega$-ERG $(m=2, M=3)$ when three lowest-energy states are kept after each iteration/sweep. RHF molecular orbitals are ordered according to their energies. The number of target states is $m=2$. See text for details. 


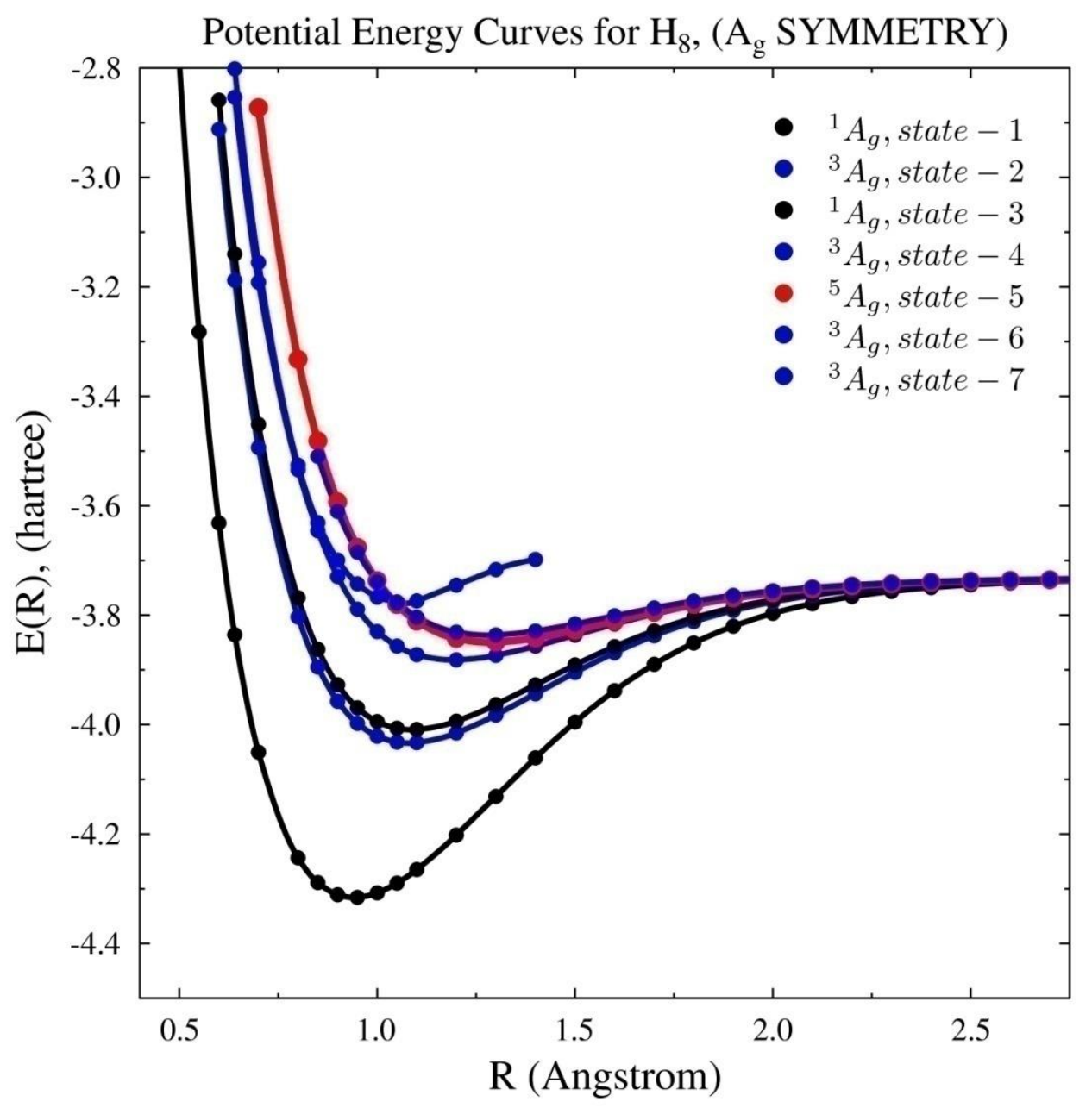

Figure 4. $\mathrm{H}_{8}$ (FCI, STO-3G, $\mathrm{A}_{\mathrm{g}}$-states). Only seven lowest-energy potential energy curves are displayed (black-circles represent singlets, blue-circles represent triplets, and red-circles represent quintuplets). RHF molecular orbitals have been used for FCI calculations (See text). 

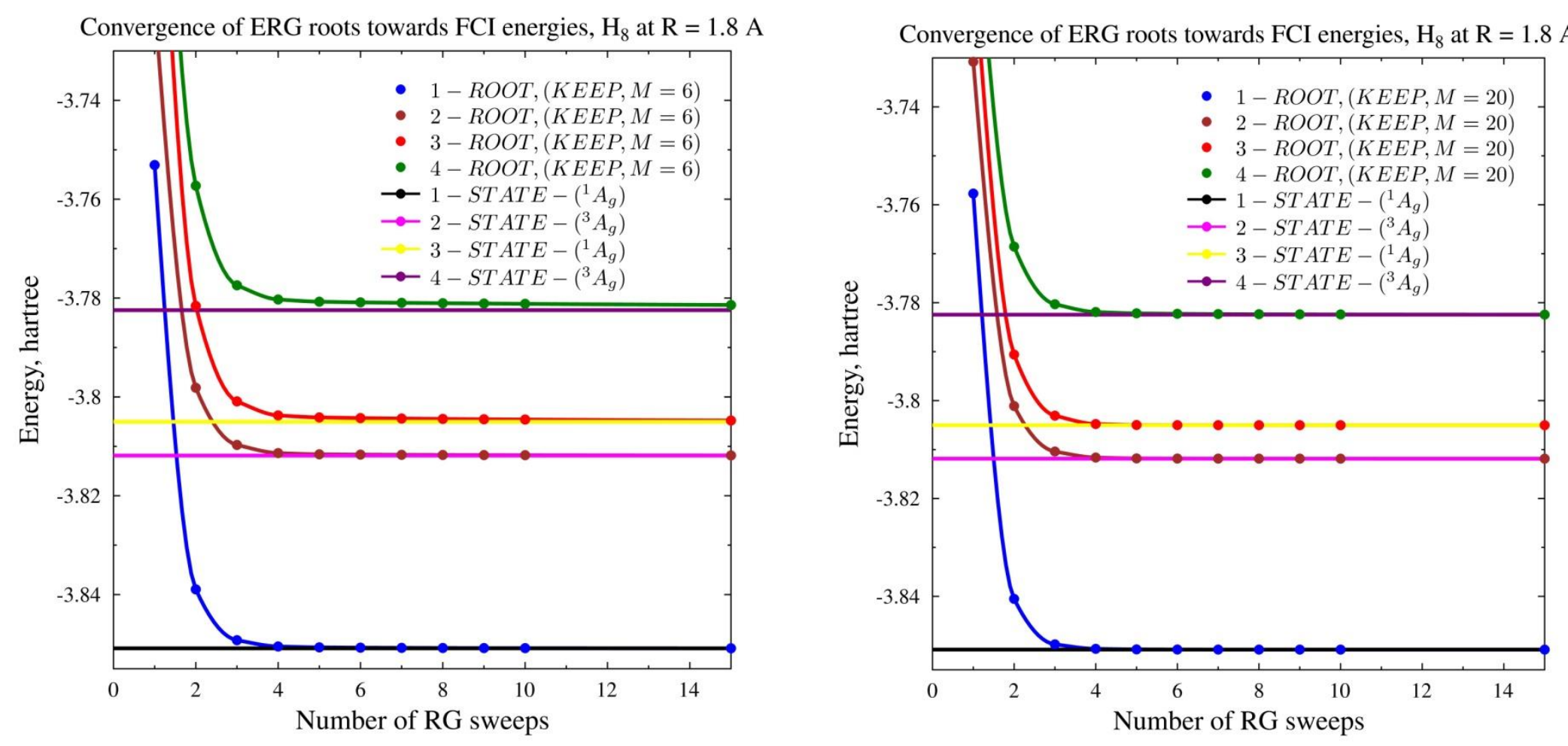

Figure 5. $\mathrm{H}_{8}(\mathrm{R}=1.8 \AA)$. Comparison of total energy convergence rates (Ag-states) for two cases: (Left-panel) $\Omega$-ERG $(m=4, M=6)$ when only six lowest-energy states are kept after each iteration/sweep; (Right-panel) $\Omega$-ERG( $m=4, M=20)$ when twenty lowestenergy states are kept after each iteration/sweep. The number of target states is $m=4$. RHF molecular orbitals are ordered according to their energies. See text for details. 

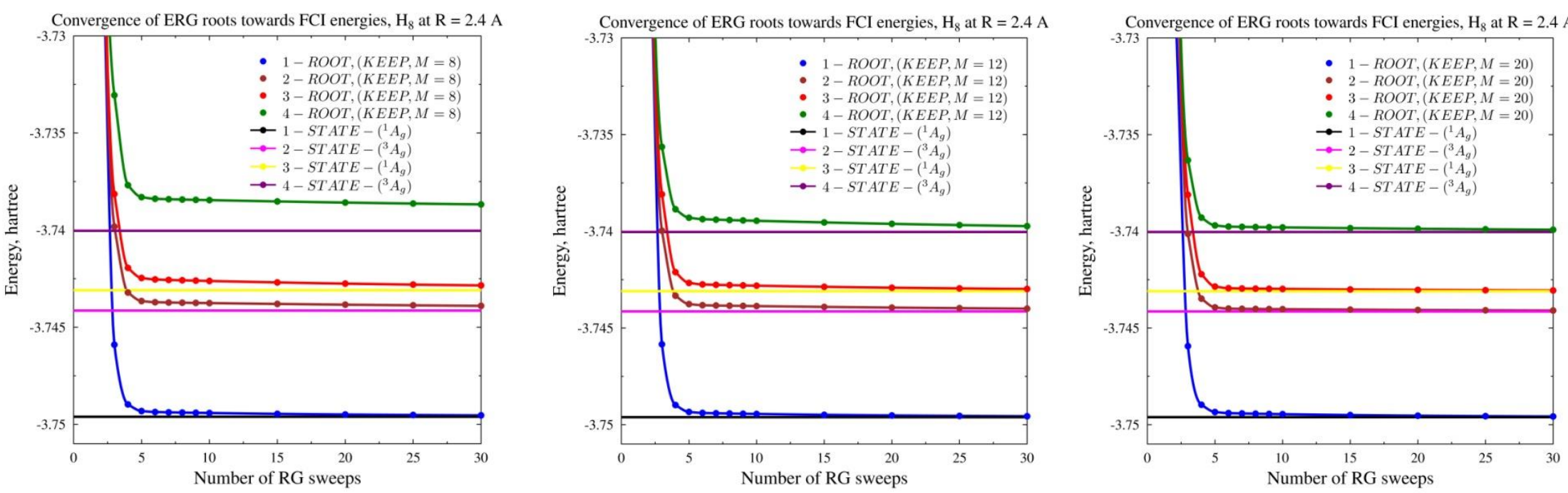

Figure 6. $\mathrm{H}_{8}\left(\mathrm{R}=2.4 \AA\right.$, $\mathrm{A}_{\mathrm{g}}$-states). Comparison of $\Omega$-ERG $(m=4, M)$ energy convergence rates for three cases: (Left-panel) only eight lowest-energy ERG roots $(M=8)$ are kept after each iteration/sweep; (Middle-panel) only twelve lowest-energy ERG states $(M=12)$ are kept after each iteration/sweep; (Right-panel) only twenty lowest-energy ERG states $(M=20)$ are kept after each iteration/sweep. RHF molecular orbitals are ordered according to their energies. See text for details. 

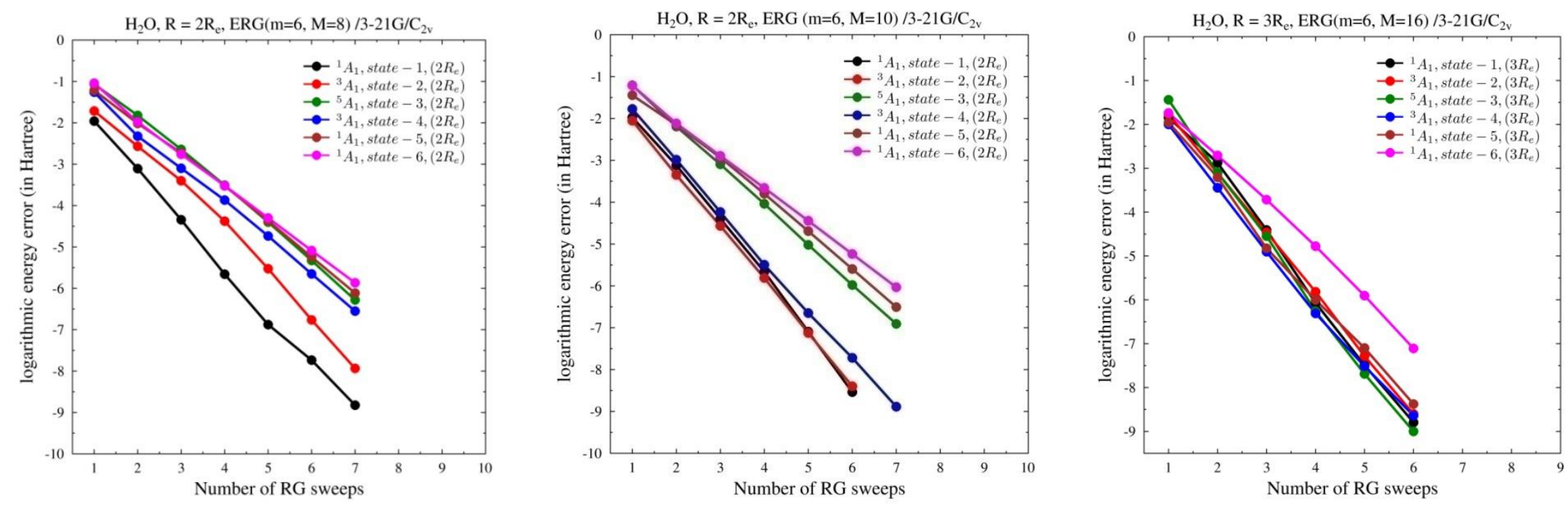

Figure 7. $\mathrm{H}_{2} \mathrm{O}$ (3-21G, $\mathrm{A}_{1}$-states). Comparison of logarithmic energy error of $\Omega$-ERG $(m=6, M)$ energies versus the number of RG sweeps for three cases: (Left-panel) only eight lowest-energy ERG roots $(M=8)$ are kept after each iteration/sweep at 2Re; (Middlepanel) only ten lowest-energy ERG states $(M=10)$ are kept after each iteration/sweep at $2 \mathrm{R}_{\mathrm{e}}$; (Right-panel) sixteen lowest-energy ERG states $(M=16)$ are kept after each iteration/sweep at $3 \mathrm{R}_{\mathrm{e}}$. Molecular orbitals in FCI calculations are taken from 2-state-averaged CASSCF $[8,6]$-calculations. See text for details. 


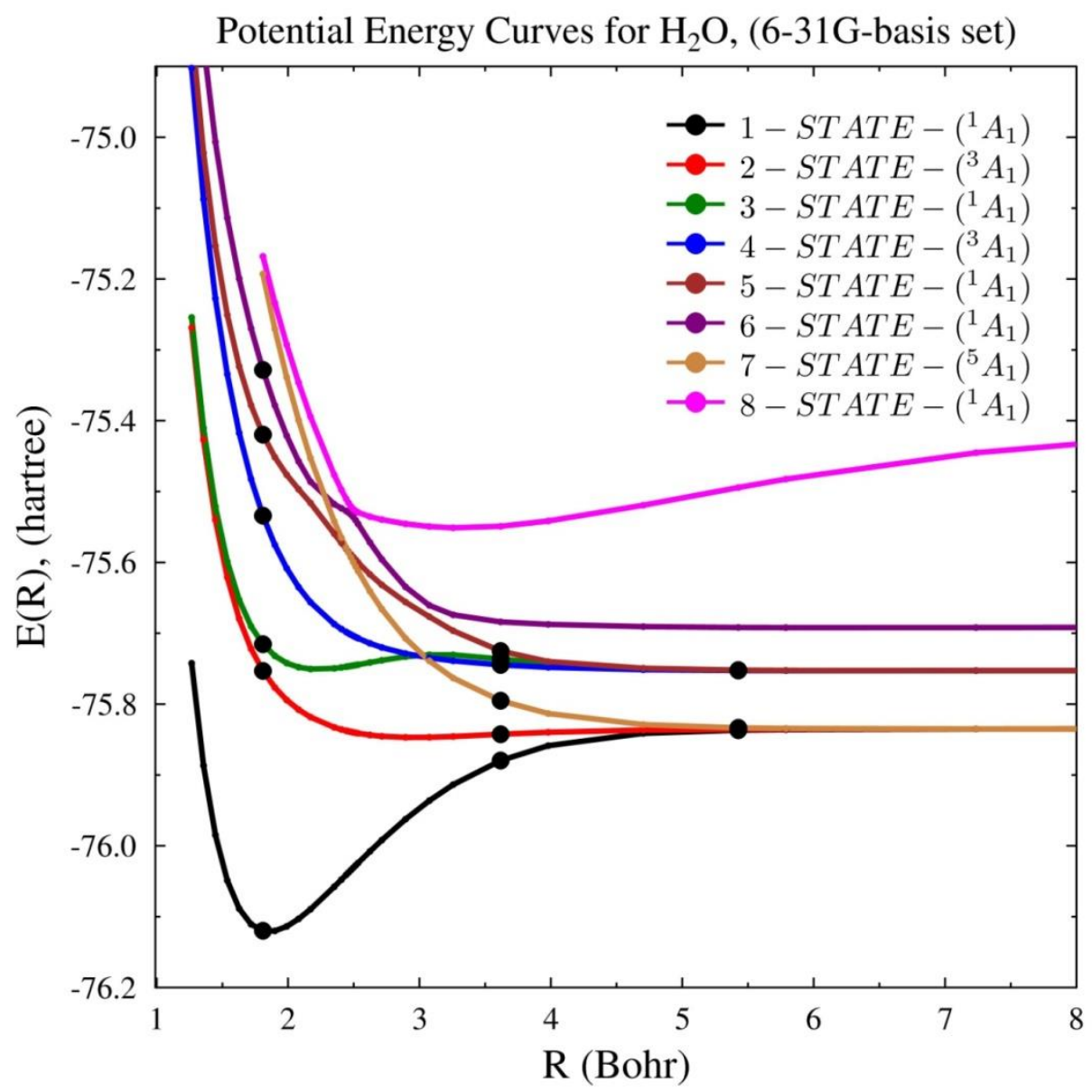

Figure 8. $\mathrm{H}_{2} \mathrm{O}$ (FCI, 6-31G, $\mathrm{A}_{1}$-states). Potential Energy Curves for lowest-energy states. Molecular orbitals for FCI calculations are taken from 2-state-averaged CASSCF[8,6]-valenceactive-space calculations. Solid circles represent data points obtained from the $\Omega$-ERG $(m, M)$ method (See text for details). 

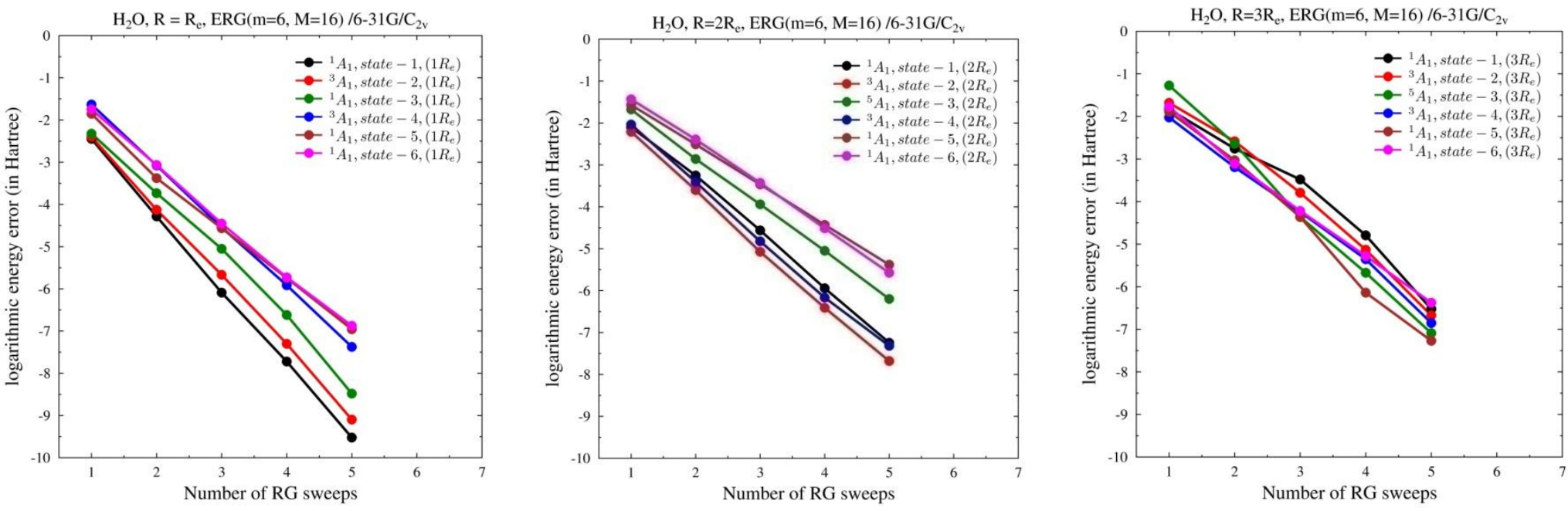

Figure 9. $\mathrm{H}_{2} \mathrm{O}$ (6-31G, $\mathrm{A}_{1}$-states). Comparison of logarithmic energy error of $\Omega$-ERG $(m=6, M=16)$ versus the number of RG sweeps for three cases: (Left-panel) at $\mathrm{R}_{\mathrm{e}}$; (Middle-panel) at $2 \mathrm{R}_{\mathrm{e}}$; (Right-panel) at $3 \mathrm{R}_{\mathrm{e}}$. Molecular orbitals in these calculations are taken from 2-state-averaged CASSCF[8,6] valence-active-space calculations. See text for details. 